\title{
ARTICLE OPEN Notch signaling represents an important checkpoint between follicular T-helper and canonical T-helper 2 cell fate
}

\author{
Mark Dell'Aringa ${ }^{1,2}$ and R. Lee Reinhardt ${ }^{1,3}$
}

Type-2 immunity is regulated by two distinct CD4+ T-cell subsets. T follicular helper (Tfh) cells are required for humoral hallmarks of type-2 inflammation. T-helper type-2 (Th2) cells orchestrate type-2 inflammation in peripheral tissues, such as the lung and intestine. Given the importance of Notch signaling in the establishment of other CD4+ T-helper cell subsets, we investigated whether canonical Notch activation could differentially impact Tfh and Th2 cell fate during the induction of type- 2 immunity. These studies show that Tfh cell, but not Th2 cell, generation and function is reliant on Notch signaling. While early Tfh cell specification is influenced by functional Notch ligands on classical dendritic cells, functional Notch ligands on cells other than dendritic cells, T cells, B cells, and follicular dendritic cells are sufficient to achieve full Th cell commitment. These findings identify Notch signaling as an early lineage-determining factor between Tfh and Th2 cell fate.

Mucosal Immunology (2018) 11:1079-1091; https://doi.org/10.1038/s41385-018-0012-9

\section{INTRODUCTION}

Type- 2 immunity and diseases associated with allergic inflammation afflict roughly 3-4 billion people worldwide, and CD4+ follicular T-helper (Tfh) cells and T-helper type-2 (Th2) cells are critical modulators of these responses., ${ }^{1,2}$ Tfh and Th2 cells orchestrate type-2 immunity through the production of type-2 cytokines. ${ }^{3}$ Interleukin (IL)-4 from Tfh cells initiates B-cell classswitching to $\lg \mathrm{E}$ and $\lg \mathrm{G} 1$, and plays a role in the selection of high affinity antibodies. ${ }^{4,5}$ Th2 cells produce IL-4, IL-5, and IL-13, and mediate their effector function in the periphery by recruiting innate immune cells to mucosal tissues to support epithelial barrier function and integrity. Although much is known regarding the canonical signaling pathways used by Th2 cells to produce IL-4 and IL-13, comparatively little is understood with respect to how Tfh cells produce IL-4. ${ }^{3}$ Furthermore, whether IL-4-producing Tfh and Th2 cells possess distinct developmental programs or share a common progenitor remains an area of continued interest in the development of type-2 immunity.

Tfh cells become competent to produce IL- 4 protein in the absence of IL-4, 4,6 and these cells express significantly lower amounts of GATA-3 messenger RNA (mRNA) and protein compared to conventional IL-4-expressing Th2 cells. ${ }^{6,7}$ As such, the canonical signaling pathways used by Th2 cells to achieve type-2 cytokine expression do not appear to be required for Tfhmediated IL-4 production. ${ }^{3}$ Recent studies have identified the $3^{\prime}$ CNS2 enhancer of the il4 locus as being essential for IL-4 production by Tfh cells. Deletion of this region abrogates Tfhmediated IL-4 production and IgE class-switching. ${ }^{8,9}$ The CNS2 region harbors binding sites for various transcription factors including multiple sites for RBP-J, an important regulator of Notchmediated signaling. ${ }^{10}$ Despite binding to the $3^{\prime}$ enhancer, the importance of Notch/RBP-J in IL-4 production by Tfh cells has been questioned. ${ }^{8}$ However, the fact that RBP-J-deficiency in CD4
+ T cells impacts IgE and IgG1 production along with the known function of RBP-J in CNS2-driven IL-4 expression by memory CD4 $+T$ cells and IL-4-expressing iNKT cells suggests that a Notchdependent mechanism may play a more important role in IL-4 production by Tfh cells than previously appreciated. ${ }^{10}$

Although Notch signaling has been studied extensively in other T-helper cell subsets, its role in Tfh cells is only starting to be elucidated. Formal evidence that Notch is playing a more general role in Tfh cell-mediated immunity comes from recent work showing that deletion of Notch1 and Notch2 in CD4+T cells prevents the generation of Tfh cells. ${ }^{11}$ The intrinsic nature of Notch signals being required for Tfh cell lineage commitment parallels findings in other T-helper cell subsets where Notch signaling facilitates cell fate choices. ${ }^{12}$ Early studies proposed that distinct Notch ligands on dendritic cells could differentially instruct the development of specific T-helper cell subsets. ${ }^{13,14}$ Although the instruction model has been challenged, ${ }^{15,16}$ this seminal work laid the foundation to explore the nature of Notch ligands in T-helper cell differentiation and function. As a result, new, non-instruction-based models have emerged. These models advocate that Notch signaling serves as an unbiased amplifier helping to stabilize the cytokine profile already being established in differentiating CD4+ T cells. ${ }^{17,18}$

Using parasitic helminth infection as a robust model of type-2 immunity, we report that Notch signaling in CD4+ T cells is required for Tfh cell generation, IL-4 production, and IgE classswitching. The defect is specific to Tfh cells as Th2-mediated immunity, worm clearance, and IL-4 expression remained normal in the absence of Notch signaling. Furthermore, an unbiased approach to eliminate all functional Notch ligands using conditional deletion of the E3 ubiquitin ligase Mind bomb1 (Mib1) in dendritic cells, B cells, T cells, and follicular dendritic cell (FDC) shows that the source of Notch ligands driving Tfh cell

\footnotetext{
${ }^{1}$ Department of Biomedical Research, National Jewish Health, Denver, CO 80206, USA; ${ }^{2}$ Department of Immunology, Duke University Medical Center, Durham, NC 27710, USA and ${ }^{3}$ Department of Immunology and Microbiology, University of Colorado Anschutz Medical Campus, Aurora, CO 80045, USA

Correspondence: R Le Reinhardt (ReinhardtL@NJHealth.org)
}

Received: 4 August 2017 Revised: 21 December 2017 Accepted: 9 January 2018

Published online: 21 February 2018 
differentiation can be distinct from the cells presenting or harboring cognate antigen during a prototypical type-2 immune response.

\section{RESULTS}

Deletion of Notch receptors in T cells results in abrogated humoral immunity but normal Th2-mediated immune hallmarks

To address the role of Notch signaling in the development of IL-4producing Th2 and Tfh cells during a type- 2 immune response, we made use of IL-4 reporter mice (IL $4^{4 \mathrm{get}}$ ), where Notch receptors are conditionally deleted in all T cells $\left(\mathrm{CD} 4^{\mathrm{cre}}\right.$ Notch $\left.1 / 2^{\mathrm{fl} / \mathrm{fl}}\right){ }^{19,20}$ The IL $4{ }^{4 g e t}$ reporter background allows for IL-4 cytokine mRNA competency to be visualized through the production of green fluorescent protein (GFP).

To evaluate type- 2 immunity, IL $4^{4 g e t}$ Notch $1 / 2^{\text {fl/fl }}$ (wild-type) and IL $4^{4 \text { get }} C D 4^{\text {cre }}$ Notch $1 / 2^{\text {fl/fl }}$ (T cell-specific, Notch-deficient) mice were infected with the helminth Nippostrongylus brasiliensis. Intestinal worm burden, lung eosinophilia, and serum lgE were assessed 9 days post infection (Fig. 1). In this model, defects in lung eosinophilia and helminth clearance are reflective of impaired Th2-mediated peripheral immunity, while defects in IgE production signify a defect in Tfh-mediated, type-2 humoral responses. ${ }^{6,21}$ Similar to wild-type mice, IL $4{ }^{4 g e t} C D 4^{\text {cre }}$ Notch $1 / 2{ }^{\mathrm{f} / / f l}$ mice mounted a productive type- 2 response in the periphery, as worms were cleared from the intestine and significant eosinophilia was observed in the lung (Fig. 1a, b). In contrast, mice lacking Notch1 and Notch2 on T cells exhibited significantly decreased IgE levels relative to wild-type animals (Fig. 1c). These data indicate that the absence of Notch signaling in T cells has little impact on Th2-mediated immune hallmarks in the periphery but a significant impact on Tfh-mediated immunity. This was not due to a defect in overall CD4+ T-cell numbers, as mice lacking Notch receptors on their $T$ cells exhibited an overall increase in CD4+ T cells in both the periphery (Supplementary Fig. S1a) and the draining lymph nodes (Supplementary Fig. S1b) after helminth infection.
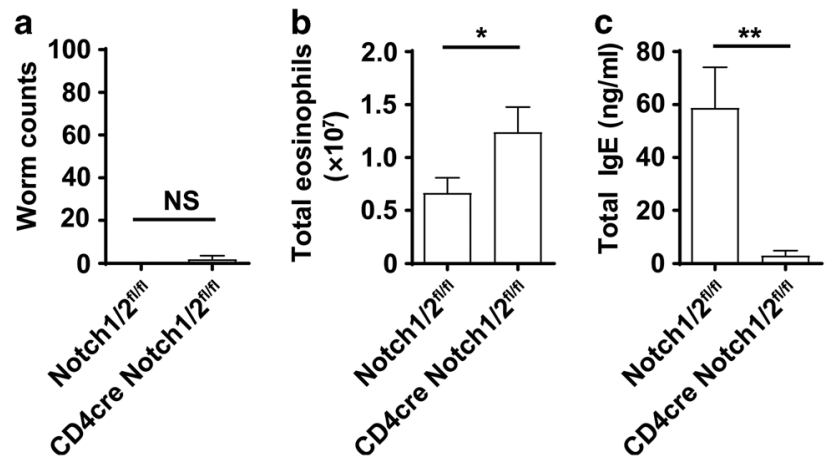

Fig. 1 Notch signaling in $\mathrm{CD} 4+\mathrm{T}$ cells is required for humoral immunity but is dispensable for Th2-mediated type-2 immune hallmarks. IL $4^{4 \text { get }}$ Notch $1 / 2^{\mathrm{fl} / \mathrm{fl}}$ and $\mathrm{IL} 4^{4 \mathrm{get}} \mathrm{CD} 4^{\text {cre }}$ Notch $1 / 2^{\mathrm{fl} / \mathrm{fl}}$ mice were infected with $N$. brasiliensis. Lung, mediastinal lymph node, and serum were harvested 9 days post infection for analysis. a Intestinal adult worm counts 9 days post infection of IL $4^{4 g e t}$ Notch $1 /$ $2^{\mathrm{f} / \mathrm{fl}}(n=13)$ and IL4 $4^{4 \mathrm{get}} \mathrm{CD} 4^{\mathrm{cre}}$ Notch $1 / 2^{\mathrm{fl} / \mathrm{fl}}(n=10)$ mice. b Graph shows the number of Siglec-F+, GFP + eosinophils of IL $4^{4 \text { get }}$ Notch1/ $2^{\mathrm{f} / \mathrm{fl}}(n=13)$ and $\mathrm{IL} 4^{4 \mathrm{get}} \mathrm{CD} 4^{\text {cre }}$ Notch $1 / 2^{\mathrm{fl} / \mathrm{fl}}(n=10)$ mice. c Total serum lgE of $\mathrm{IL} 4^{4 \text { get }}$ Notch $1 / 2^{\mathrm{f} / \mathrm{fl}} \quad(n=13)$ and IL4 ${ }^{4 \text { get }} \mathrm{CD} 4^{\text {cre }}$ Notch $1 / 2^{\mathrm{f} / \mathrm{fl}}(n=10)$ mice. Error bars represent $+/$ - SEM. Data shown is combined from three independent experiments with $n=3-5$ mice per group per experiment. ${ }^{*} P<0.05$, ${ }^{*} P<0.01$, (unpaired twotailed $t$-test)
T-cell-specific deletion of Notch receptors leads to a selective reduction in IL-4 competent CD4+ T cells in the lymph nodes compared to the lungs after helminth infection

Given that we observed normal worm clearance and eosinophilia but reduced IgE production in mice lacking Notch in T cells, we hypothesized that type-2 cytokine competency (mRNA expression) in Notch-deficient Th2 cells would remain intact, while IL-4competent Tfh cell number or function would be compromised. In support, mice that lacked expression of Notch in T cells showed normal numbers of IL-4 competent cells (IL4 ${ }^{4 \text { get }}$ GFP-reporter positive; IL-4 mRNA) in the lung, despite a decreased percentage of IL-4 competency within the total CD4+ T-cell pool (Fig. 2a). This is consistent with the higher number of CD4+T cells found in the lungs of these mice after infection (Supplementary Fig. S1a). Despite normal Th2 cell generation, IL-4-expressing CD4+T cells were significantly reduced in both percentage and number in the lung-draining mediastinal lymph nodes when Notch1 and Notch2 were deleted from T cells (Fig. 2b).

These findings were further supported by histological analysis of IL-4-expression in the lung and lymph nodes. CD4+ GFPreporter staining in the lung was similar between both wild-type mice and animals lacking Notch1 and Notch2 in T cells (Fig. 3a). The lack of GFP-reporter staining in tissue sections of mediastinal lymph nodes taken from IL4 ${ }^{4 g^{\mathrm{get}}} \mathrm{CD} 4^{\mathrm{cre}} \mathrm{Notch} 1 / 2^{\mathrm{f} / \mathrm{fl}}$ mice confirms the paucity of IL-4 -expressing T cells in both the paracortex (IgD-) and B-cell follicles $(\lg \mathrm{D}+$ ) of the lymph nodes when Notch signaling is absent (Fig. 3b). In sum, these data are consistent with a more pronounced role for Notch signaling in establishing type-2 cytokine competency in Tfh cells residing in the lymph nodes compared to lung-resident, canonical Th2 cells.

Tfh cell generation, IL-4 expression, and germinal center B-cell numbers are significantly reduced in mice that lack Notch in T cells

Reduced serum IgE and IL-4 expression in the follicles of the mediastinal lymph nodes suggested that Tfh cell generation and/or function could be impacted by Notch-deficiency in T cells. In support, Tfh cells isolated from helminth-infected mice showed significantly increased Notch1 and Notch2 receptor expression relative to non-Tfh cells (Supplementary Fig. S2a). To directly investigate the necessity of Notch in Tfh cell generation, mediastinal lymph nodes were isolated from $\mathrm{IL} 4^{4 \mathrm{get}} \mathrm{CD} 4^{\text {cre }}$ Notch $1 / 2^{\mathrm{fl} / \mathrm{fl}}$ and IL $4^{4 \text { get }}$ Notch $1 / 2^{\mathrm{fl} / f l}$ mice 9 days after infection with $N$. brasiliensis, and $C D 4+T$ cells were analyzed for canonical Tfh cell markers CXCR5 and PD-1 (Fig. 4a). T-cell Notch-deficiency results in a significant impairment in both the percentage and number of Th cells within the CD4+ T-cell compartment (Fig. 4a). This result was confirmed as Notch-deficient CD4+ T cells from the mediastinal lymph nodes showed a marked deficit in expression of the Tfh cell lineage-determining factor BCL6 by percent and mean fluorescent intensity (MFI) (Supplementary Fig. S3a). GATA3, a key lineagedetermining factor for Th2 commitment, was not significantly changed in percentage or MFI (Supplementary Fig. S3b).

Regarding an effect on IL-4 mRNA expression, in the few CXCR5+, PD1+ cells phenotypically resembling Tfh cells generated in infected IL $4{ }^{4 \mathrm{get}} \mathrm{CD} 4^{\text {cre }}$ Notch $1 / 2{ }^{\mathrm{f} / \mathrm{fl}}$ mice, we observed a tenfold reduction in GFP (IL-4) reporter expression compared to Notchsufficient T cells (Fig. 4b). This deficiency in Tfh cells and Tfh-derived IL-4 corresponded to impaired germinal center B-cell generation, an event largely dependent on Tfh cells in this model (Fig. 4c). Altogether, these data identify Notch signaling as a key component in Tfh cell generation and function during type- 2 immunity.

Because the deletion of both Notch 1 and 2 resulted in an almost complete loss of Tfh cells, it was difficult to fully assess the role of Notch signaling in IL-4 expression by Tfh cells. To circumvent this issue, we made use of an intermediate phenotype observed in mice that lack Notch1 but retain one allele of Notch2. This led to an intermediate loss of Th cells compared to Notch-sufficient and 
a

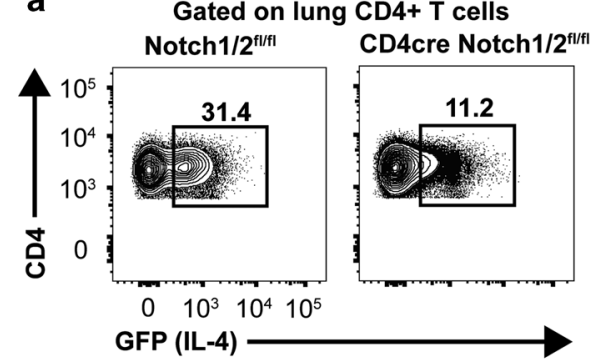
Notch $1 / 2^{\text {fl/fl }}$

FP (IL-4)
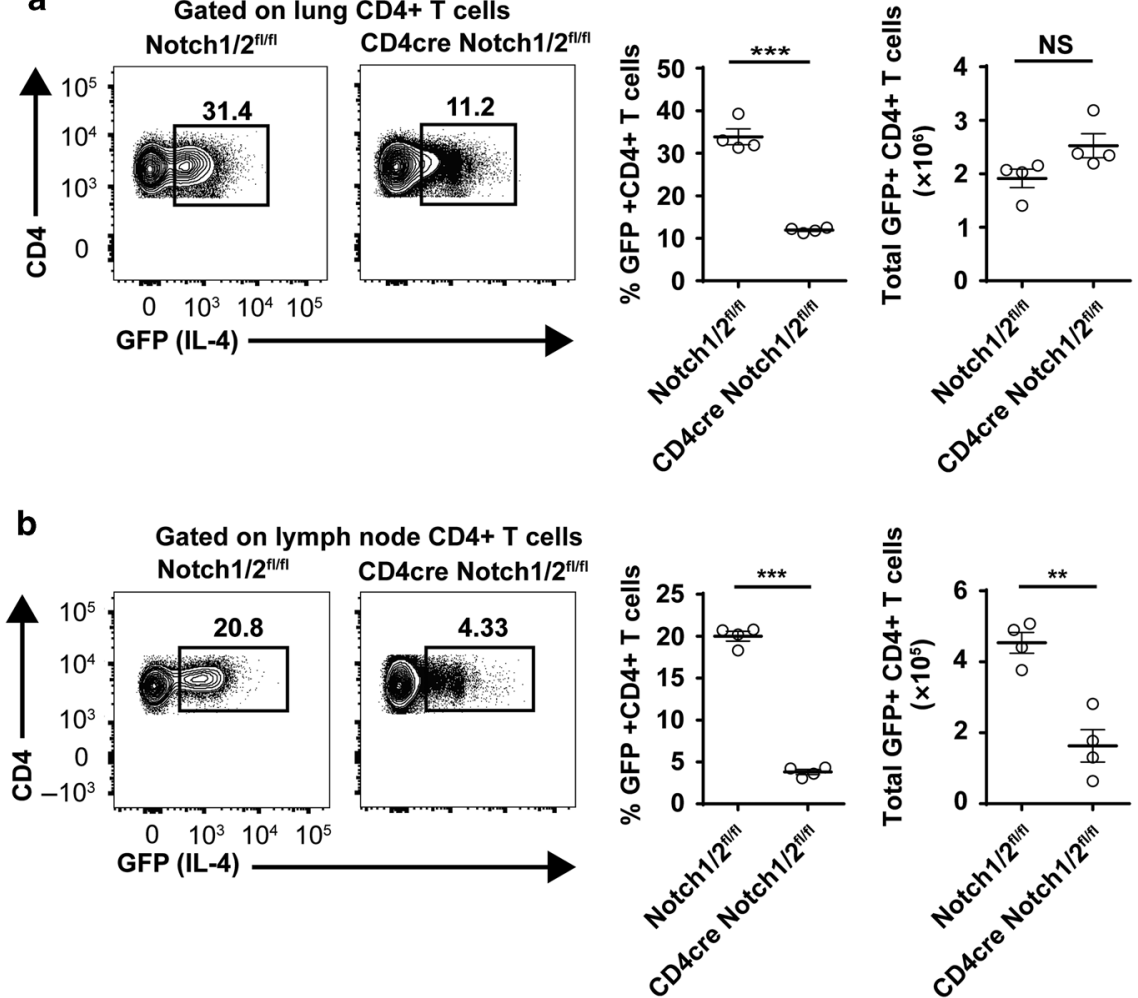

Fig. 2 Deletion of Notch receptors on T cells results in reduced IL-4 competency in CD4+ T residing in the lymph node cells but not lung. IL $4{ }^{4 g e t}$ Notch $1 / 2^{\mathrm{f} / / \mathrm{fl}}(n=4)$ and IL4 ${ }^{4 g e t} \mathrm{CD} 4{ }^{\mathrm{cre}}$ Notch $1 / 2^{\mathrm{f} / \mathrm{fl}}(n=4)$ mice were infected with $N$. brasiliensis. Mediastinal lymph nodes and lung were harvested 9 days later and prepared for flow cytometry. a Representative contour plot gated on total CD4+ T cells in the lung and $\mathbf{b}$ lymph node. Gates indicate the percent of IL-4 competent cells. Graphs show percent and total number of GFP + CD4+T cells. Error bars represent $+/$ - SEM. Data is representative of four independent experiments with $n=3-5$ per group. ${ }^{* *} P<0.01,{ }^{* *} P<0.001$ (unpaired two-tailed $t$-test)

Notch1/2-deficient CD4+T cells (Fig. 5a). Importantly, the Tfh cells generated in the absence of Notch1 still exhibited a significant impairment in IL-4 mRNA expression (Fig. 5b). In line with these results, elimination of Notch1 alone in $\mathrm{T}$ cells caused a moderate reduction in germinal center B-cell generation (Fig. $5 \mathrm{c}$ ).

To further investigate the role of Notch signaling in the expression of IL-4 by Tfh cells, we employed the use of a gamma secretase inhibitor (GSI) to inhibit Notch signaling in already differentiated Tfh cells. IL4 ${ }^{4 g e t / K N 2}$ mice immunized with ovalbumin (OVA) precipitated in alum generate abundant Tfh cells by 7-10 days after subcutaneous immunization. ${ }^{4}$ Using this model, OVA immunized mice were given injections of GSI or a mock injection on days 7,8 , and 9 post immunization. Mice that received the GSI had a reduction in both their percentage and number of Tfh cells (Supplementary Figs. S4a and S5a). Importantly, IL-4 protein production by Tfh cells in mice that received GSI was significantly reduced compared to mice that received a mock injection (Supplementary Fig. S4b). Thus, both the absence of Notch 1 and the blocking of Notch signaling in established Tfh cells results in reduced Tfh cell numbers and IL-4 expression among the remaining Tfh cells.

BCL6, BATF, IRF4, and cMaf all play important roles in Th cell differentiation. ${ }^{21-26}$ Many of these factors also have been suggested to play a role in IL-4 expression by Tfh cells. ${ }^{21,22}$ To assess if Notch signaling influences these Tfh lineage-factors, we again generated Tfh cells and gave GSI or a mock injection on days 7, 8, and 9 before performing intracellular transcription factor staining. GSI treatment led to decreased c-Maf expression among $\mathrm{BCL} 6+, \mathrm{PD} 1^{\text {high }}$ Tfh cells (Supplementary Figs. S5b and S6b), while the expression of IRF4 and BATF was not significantly changed (Supplementary Figs. S5c, d and S6c, d). C-Maf is known to regulate IL-4 expression in Th2 cells, but its role in Tfh-driven IL-4 expression is unclear. $^{26}$

Over-expression of Notch intracellular domain increases recent IL4 protein production in Th cells

The data demonstrate that Notch-deficiency in T cells leads to impaired Tfh cell generation and an impaired ability to establish IL-4 mRNA competency within those cells. To better uncouple the role of Notch in Tfh generation from its role in Tfh-derived IL-4 production, we investigated whether additional Notch signaling would lead to increased IL-4 protein production in Tfh cells. To do this, IL $4^{\mathrm{KN} 2}$ reporter mice, ${ }^{27}$ which report recent IL-4 protein production through the expression of human $C D 2$ at the cell surface, were crossed with a tamoxifen-inducible GFP-NICD fusion reporter line. ${ }^{28}$ In this IL4 ${ }^{\mathrm{KN2}} \mathrm{ER}^{\text {cre }}$ Rosa26 $6^{\text {Stop-flox-NICD-GFP }}$ gain-of-function system, it is possible to directly compare IL-4 protein production in Tfh cells experiencing endogenous Notch signals relative to those experiencing increased Notch signaling in the same lymph node based on the presence or absence of GFP expression.

IL4 ${ }^{\mathrm{KN2}} \mathrm{ER}^{\text {cre }}$ Rosa26 $6^{\text {Stop-flox-NICD-GFP }}$ mice were treated with tamoxifen 3 days prior to $N$. brasiliensis infection, and mediastinal lymph nodes and lungs from mice were analyzed 9 days post infection (Fig. 6a). IL-4 protein production, as marked by human CD2, was comparable between CD4+ T cells-expressing endogenous NICD (GFP negative) and those over-expressing NICD (GFP-positive) in the lung (Fig. 6b). In contrast, Tfh cells from the mediastinal lymph nodes of these same animals showed a significant twofold enhancement of IL-4 production when the GFP-NICD fusion was induced (Fig. 6C). Over-expression of NICD also led to an increase in the percentage of non-Tfh cells producing IL-4 in the lymph node (Supplementary Fig. S7a, b). 
a
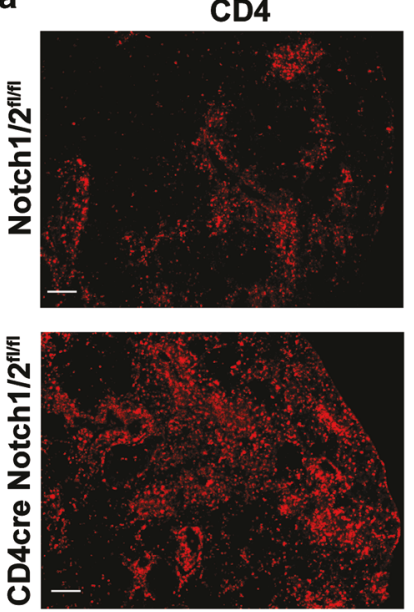

b
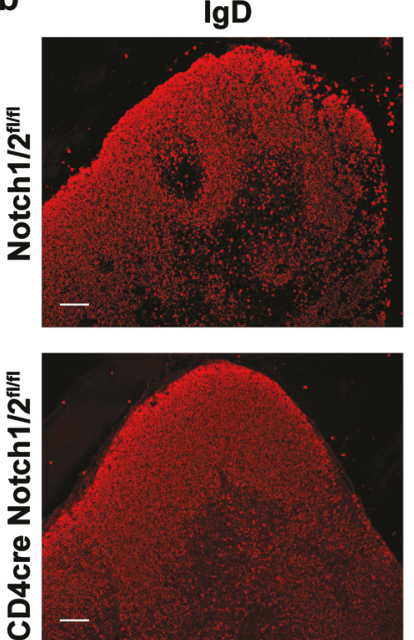

GFP
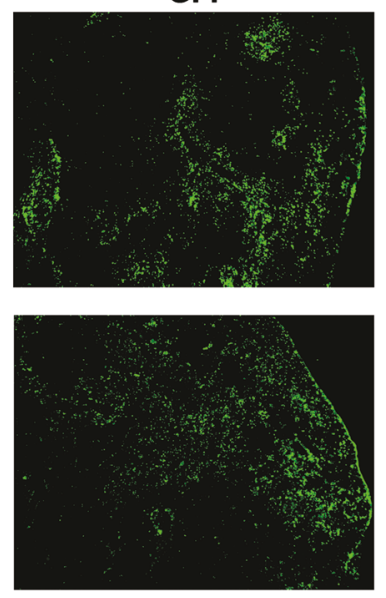

GFP
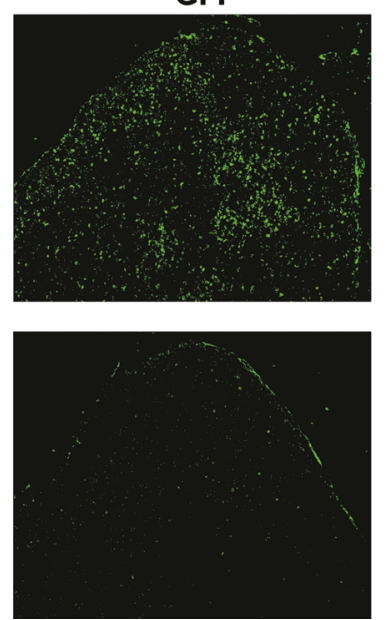
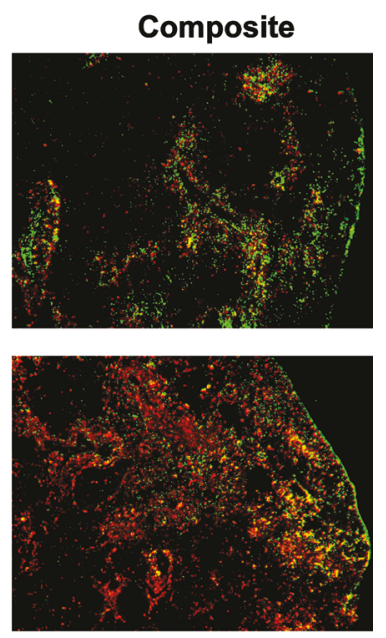

Composite
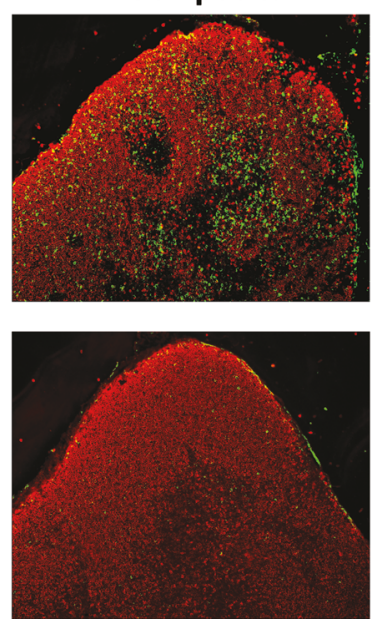

Fig. 3 Deletion of Notch1 and Notch2 on T cells results in decreased CD4+ T cell IL-4 expression in lymph node but not lung. IL4 ${ }^{4 g e t}$ Notch1/ $2^{\mathrm{f} / f l}$ and IL $4^{4 \mathrm{get}} \mathrm{CD} 4^{\text {cre }}$ Notch $1 / 2^{\mathrm{f} / f \mathrm{fl}}$ mice were infected with $N$. brasiliensis. Nine days later, lung and mediastinal lymph node were harvested, fixed, and sectioned for subsequent immunofluorescence microscopy. a Lung sections were stained for CD4 (red) and GFP (IL-4 competency; green). Channels were merged and are shown as a composite image. b Lymph node sections were stained for IgD (red) and GFP (IL-4 competency; green). A composite image is shown. Scale bars (bottom left) represent $100 \mu \mathrm{m}$. Images shown are representative of three independent experiments with $n=2-3$ per group

In addition, NICD gain-of-function also led to increased BCL6 and GATA3 expression in lymph node CD4+ T cells (Supplementary Fig. S8a, b). This finding is consistent with Notch signaling affecting GATA3 expression, as previously shown during in vitro Th2 cultures, ${ }^{19,29}$ but also highlights a role for Notch signaling in mediating BCL6+ Tfh cell generation or maintenance. ${ }^{6}$ BCL6 and GATA3 in lung CD4+ T cells was not altered in cells overexpressing NICD (Supplementary Fig. S8c, d). Altogether, these data show that Notch signaling impacts not only Tfh cell development but also IL-4 production by Tfh cells. The observation that over-expression of NICD has a more pronounced effect on IL-4 production and transcriptional regulation in Th cells compared to lung Th2 cells further supports a more selective role for Notch signaling in Th cells relative to their Th2 cell counterparts.

Functional Notch ligands on conventional dendritic cells can influence Tfh cell fate early during CD4+ T-cell differentiation, but are not required for full Tfh commitment

Although high doses of antigen can drive a Tfh cell fate in the absence of conventional dendritic cells (cDC), the initiation of Tfh cell fate often requires antigen presentation by conventional dendritic (cDC) cells in more physiologic settings. ${ }^{30-32}$ To assess whether early Tfh cell commitment required functional Notch ligands on CDC subsets, canonical Notch ligand-mediated signaling was prevented by conditionally deleting the E3 ubiquitin ligase molecule, Mind bomb1 (Mib1), in CDC. Notch signaling requires Mib1 for ligand-induced Notch signaling in vertebrates. ${ }^{33}$ Deletion in dendritic cells was achieved by CD11c-driven, Cre recombinase-mediated excision of loxP-flanked Mib1. ${ }^{34,35}$ Cre recombinase expression in $\mathrm{CDC}$ was confirmed by assessing yellow fluorescent protein (YFP) expression among CD11c+, MHCll+cells isolated from the mediastinal lymph nodes of $N$. brasiliensisinfected $C D 11 c^{\text {Cre }}{ }_{\text {Rosa }}^{\text {flox-stopYFP }}$ mice (Supplementary Fig. S9a). Mib1 deletion using the $\mathrm{CD} 11 \mathrm{c}^{\mathrm{Cre}} \mathrm{Mib}^{\mathrm{f} / \mathrm{fl}}$ system was confirmed by PCR analysis of dendritic cells isolated and sorted from the mediastinal lymph nodes 5 days after $N$. brasiliensis infection (Supplementary Fig. S9b) and bone marrow-derived DC cultures (Supplementary Fig. S9c).

To assess changes in IL-4 competency and Tfh development in the absence of Mib1 in $\mathrm{CDC}$, we crossed $\mathrm{CD} 11 \mathrm{c}^{\mathrm{Cre}} \mathrm{Mib} 1^{\mathrm{fl} / \mathrm{fl}}$ mice onto the IL $4^{4 g e t}$ reporter system. IL $4^{4 g e t} C D 11 c^{\text {Cre }}$ Mib1 ${ }^{\mathrm{fl} / \mathrm{fl}}$ (Mib1deficient $\mathrm{CDC}$ ) and IL4 ${ }^{4 \mathrm{get}} \mathrm{Mib}^{\mathrm{f} / \mathrm{fl}}$ (wild-type) mice were infected with $N$. brasiliensis. Mediastinal lymph nodes were isolated on day 
a

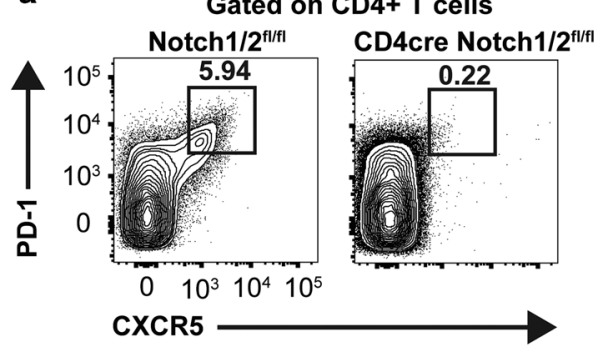

b

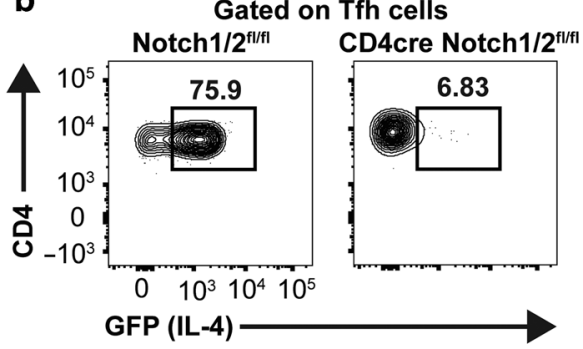

C

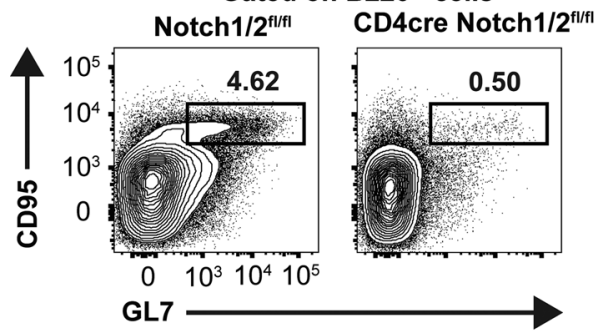

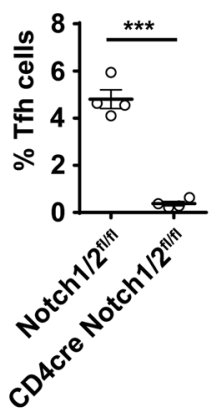
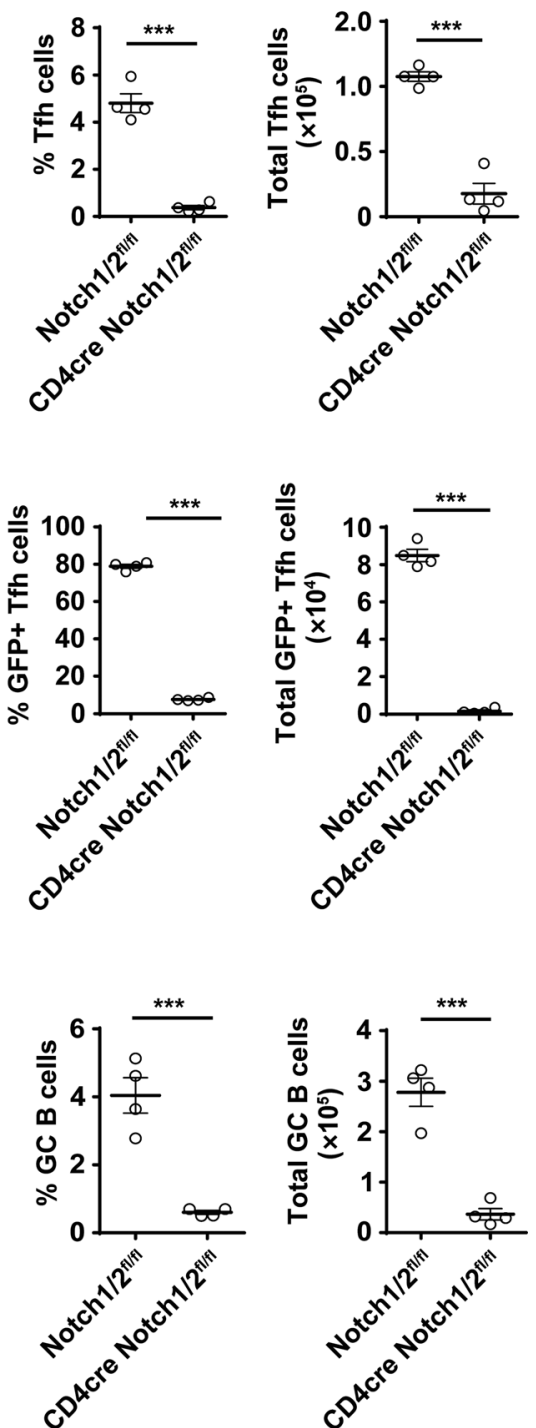

Fig. 4 Notch deficiency in T cells results in reduced Tfh cell numbers, Tfh IL-4 production, and germinal center B cells. Mediastinal lymph nodes of IL4 ${ }^{4 g e t} \operatorname{Notch} 1 / 2^{\mathrm{f} / / f l}(n=4)$ and IL $4^{4 g e t} \mathrm{CD} 4^{\text {cre }}$ Notch $1 / 2^{\mathrm{f} / \mathrm{fl}}(n=4)$ mice were collected 9 days after infection with $N$. brasiliensis. a Representative contour plots of CD4+ T cells. Gate represents the percentage of Tfh cells (PD-1+, CXCR5+). Graphs show percentage and total number of Tfh cells in indicated mice. b Representative contour plots of Tfh cells gated in panel a. Gate represents the percentage of GFP+ Tfh cells. Graphs show percent and number of IL-4 competent Tfh cells from indicated mice. c Contour plots pre-gated on B220+ cells. Gate represents germinal center B cells $(\mathrm{CD} 95+\mathrm{GL} 7+)$. Graphs show percent and total number of GC B cells within the total B220+ population. Error bars represent $+/$ - SEM. Data is representative of four independent experiments with $n=3-5$ mice per group. ${ }^{* * *} P<0.001$ (unpaired two-tailed $t$-test)

5 and day 9 post infection. On day 5, mice lacking Mib1 in CD11cexpressing cells showed a significant decrease in the percentage and total number of CD4+T cells expressing the canonical Tfh markers CXCR5+, PD-1+ (Fig. 7a). Although the percentage of Tfh cells-expressing IL-4 mRNA were similar between mice harboring Mib1-deficient CDC and wild-type CDC, the numbers of IL-4 competent Tfh cells were reduced as would be expected given the decrease in total Tfh cells (Fig. 7a, b). However, the dependence on functional Notch-ligands provided by CDC was not absolute as Tfh cell numbers and IL-4 mRNA competency were similar by day 9 of infection (Fig. 7c and Supplementary Fig. S10a, b).

The defect observed at day 5 was not due to a general priming defect as IL4 ${ }^{4 \text { get }} \mathrm{OT}-\mathrm{II}$ CD4 $+\mathrm{T}$ cells transferred into $\mathrm{CD} 11 \mathrm{C}^{\mathrm{cre}} \mathrm{MIB}^{\mathrm{fl} / \mathrm{fl}}$ mice did not have a significant defect in their proliferation compared to OT-II T cells transferred into control $M I B 1^{f l / f l}$ mice (Supplementary Fig. S11a). The transferred cells also had no defect in the expression of early activation markers (Supplementary Fig. S11b). Consistent with the previous findings in Tfh cells, OT-II T cells transferred into $\mathrm{CD} 11 \mathrm{c}^{\mathrm{cre}} \mathrm{MIB} 1^{\mathrm{f} / \mathrm{fl}}$ mice exhibited no reduction in the percentage of cells that established IL4 mRNA competency (Supplementary Fig. S11c).

Functional Notch ligands on B cells and follicular dendritic cells are not required for Tfh cell commitment Although CDCs often play an important role in the early development of Tfh cells, entry of Tfh cells into the B-cell follicles and interaction with B cells themselves appears critical for ultimate Tfh cell commitment. ${ }^{31}$ Furthermore, depletion of FDC impacts Tfh cell numbers. ${ }^{36}$ Given that functional Notch ligands on CDC were not required for Tfh cell commitment after day 5 , we pursued the idea that B cells or FDC in the follicles might be the compensatory source of these ligands as the cellular response to 
a

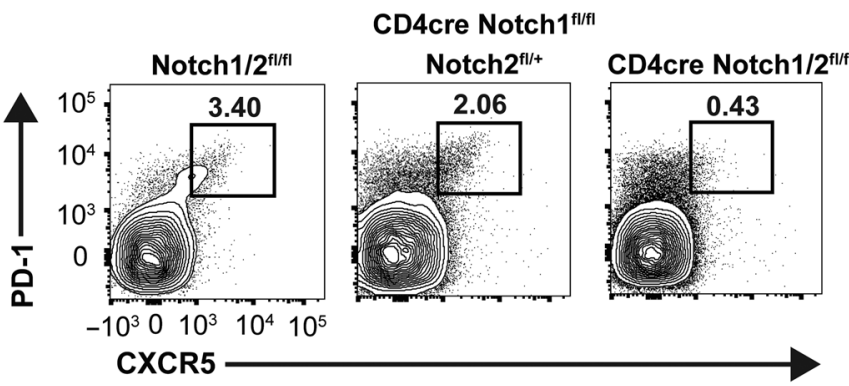

b

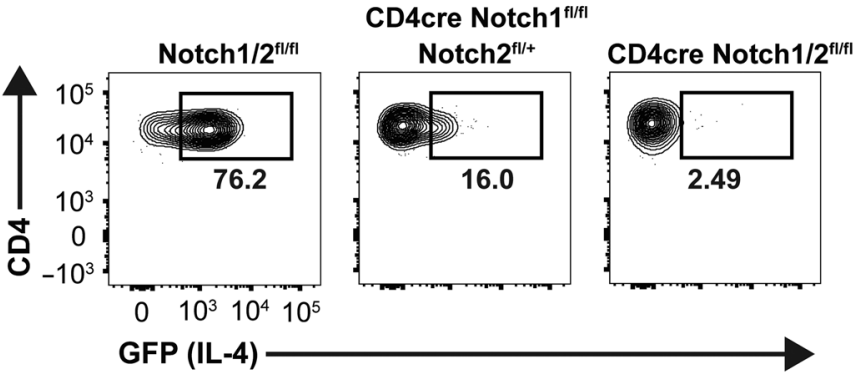

C

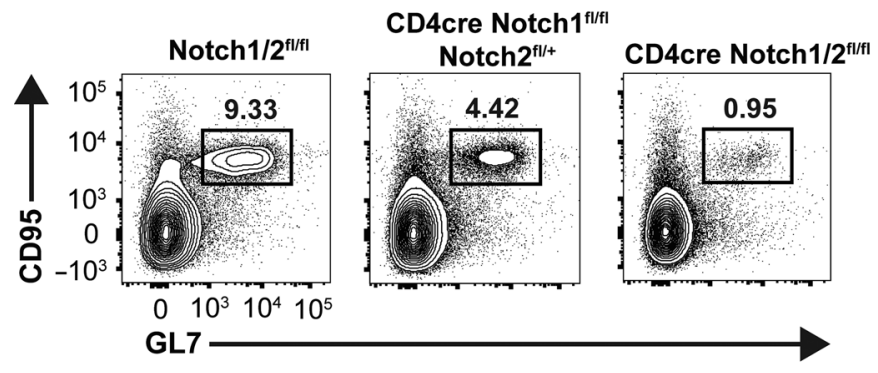

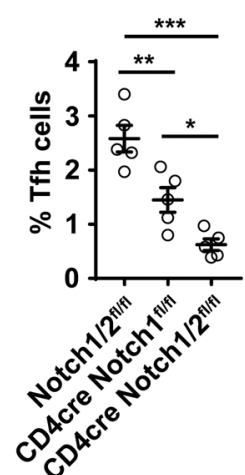
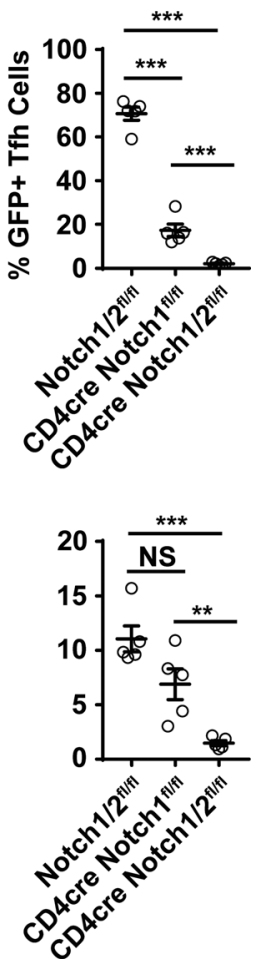

Fig. 5 Deletion of Notch1 alone results in an intermediate phenotype compared to $T$ cells deficient in both Notch1 and Notch2.

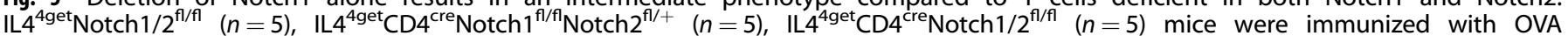
emulsified in alum in the footpad. Eight days post immunization, the popliteal lymph node was harvested for flow cytometry. a Representative contour plots of CD4+ T cells. Gate represents Tfh cells (PD-1+, CXCR5+). Graph shows the percentage of Tfh cells of total CD4 + T cells. b Representative contour plots showing of Tfh cells gated in panel a. Gate represents GFP+ Tfh cells. Graph shows the percent of IL4 competent Tfh cells from indicated mice. c Contour plots pre-gated on B220+ cells. Gate represents germinal center B cells (CD95+ GL7+). Graph shows the percent of germinal center B cells within the B220+ population. Error bars represent +/- SEM. Data is combined from two independent experiments with $n=2-3$ mice per group. ${ }^{*} P<0.05,{ }^{* *} P<0.01,{ }^{* * *} P<0.001$ (unpaired two-tailed $t$-test)

N. brasiliensis matured. To investigate this mechanism, we conditionally deleted Mib1 in B cells and FDC using a CD21driven Cre recombinase. ${ }^{37} \mathrm{CD} 21$ is confined to these two cell types in the mouse immune system, ${ }^{38}$ and the specificity of CD21-Cre recombinase activity has been confirmed in both $\mathrm{B}$ cells and FDC using this system. ${ }^{36,37}$

To confirm CD21-Cre activity in FDC and B cells in our hands, we immunized $\mathrm{CD} 21^{\text {cre }}$ Rosa26 $6^{\text {Stop-flox-YFP }}$ mice with OVA precipitated in alum at three sites in the back and collected draining axillary, brachial, and inguinal lymph nodes 5 days post injection. Similar to previous reports, Cre-activity was confirmed in $87.1 \%$ of the B cells by YFP expression (Fig. 8a). Using flow cytometry, we observed that CD21-cre-activity was highly enriched in FDCs compared to other stromal populations based on YFP expression (Fig. 8b). Using immunohistochemistry on CD21 ${ }^{\text {cre }}$ Rosa26 $6^{\text {Stop-flox- }}$
YFP mice, we confirmed CD21-cre-activity in FDC and B cells by staining for YFP in tissue sections obtained from isolated lymph nodes (Fig. 8c). YFP was co-expressed by both lgD+ (B cells) cells, as well as FDCM2 + (FDC) cells in the lgD- germinal center (Fig. 8c).

To assess whether functional Notch ligands on $B$ cells and FDC are required for Tfh commitment, IL $4^{4 \text { get }} \mathrm{CD} 21^{\mathrm{Cre}} \mathrm{Mib} 1^{\mathrm{fl} / \mathrm{fl}}$ (Mib1deficient $\mathrm{B}$ cells and FDC) or IL4 ${ }^{4 \mathrm{get}} \mathrm{Mib}^{\mathrm{fl} / \mathrm{fl}}$ (wild-type) mice were infected with $N$. brasiliensis, and mediastinal lymph nodes were isolated 9 days later. Both CXCR5+, PD1+ Tfh cell percentages and numbers were similar to mice where $B$ cells and FDC remained Mib1 competent (Fig. 9a). There was also no significant change in IL-4 mRNA competency among the Tfh cell compartment, as percentage and number of GFP expressing cells remained similar between these two groups (Fig. 9b). T-cell-specific deletion of Mib1 also had no impact on Tfh cell numbers or IL-4 potential 


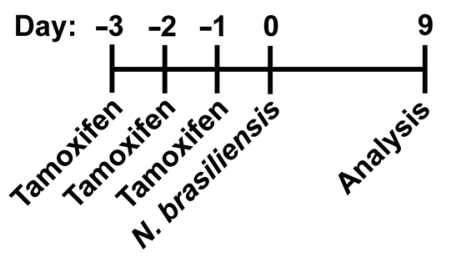
(1)

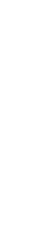

b

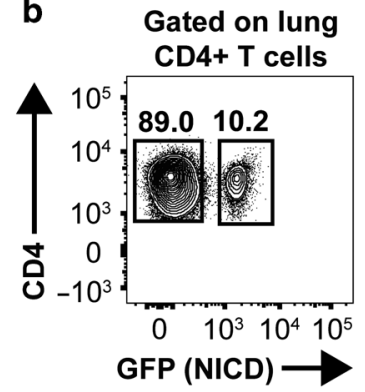

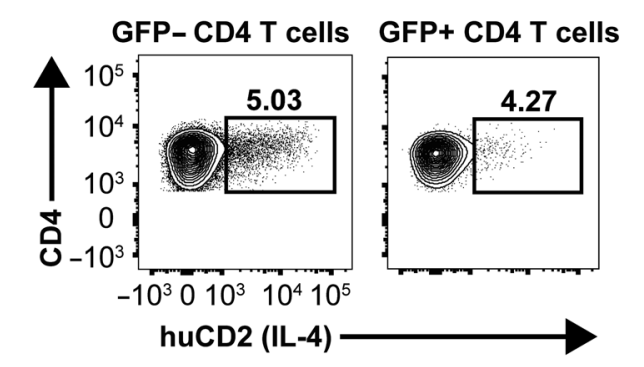

C Gated on lymph node

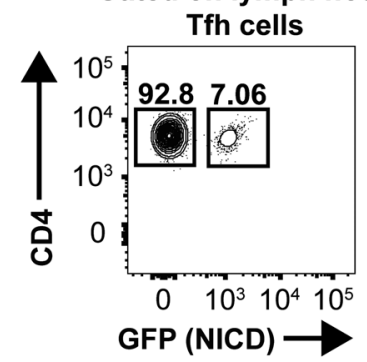

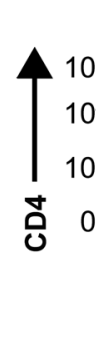

GFP- Tfh cells

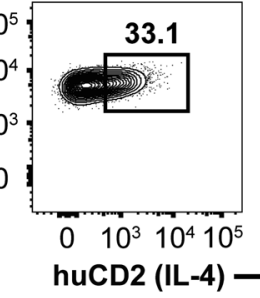

GFP+ Tfh cells

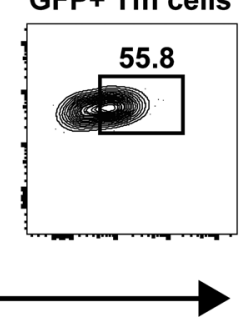

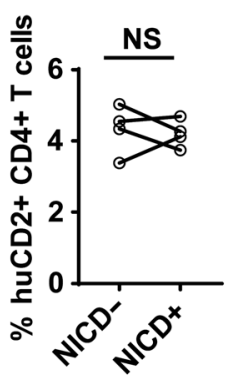

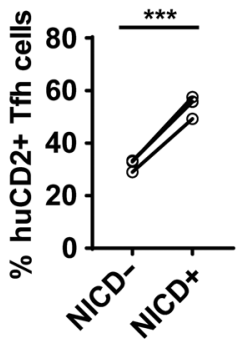

Fig. 6 Over-expression of Notch signaling leads to increased IL-4 production by Tfh cells but not lung-resident CD4+ T cells. a Diagram of experimental set-up: IL $4^{\mathrm{KN} 2} \mathrm{ER}^{\text {cre }}$ Rosa2 $6^{\text {Stop-flox-NICD-GFP }}(n=4)$ mice were injected with tamoxifen once every $24 \mathrm{~h}$ for 3 days starting on day 3. On day 0 mice were infected with $N$. brasiliensis and mediastinal lymph nodes were harvested on day 9. b Representative flow plots of CD4+ T cells isolated from the lung. Gates shown represent cells with endogenous Notch signaling (CD4+ GFP-) and cells over-expressing NICD (CD4+ GFP+). IL-4 protein (huCD2) expression was analyzed and quantified as a percent of the gated populations. c Contour plots of Tfh (PD1 + CXCR5+) cells isolated from mediastinal lymph nodes. IL-4 protein (huCD2) expression was assessed and quantified in Tfh cells with endogenous levels of Notch signaling (CD4+, PD1+, CXCR5+, GFP-) and Tfh cells over-expressing NICD (CD4+, PD1+, CXCR5+, GFP+). Error bars represent +/- SEM. Data is representative of four independent experiments with $n=3-5$ mice per group. ${ }^{* * *} P<0.001$ (paired twotailed $t$-test)

(Supplementary Fig. S12a, b). To summarize, functional Notch ligands on CDCs influence early Tfh cell fate and cytokine competency. However, functional ligands on non-hematopoietic cells other than FDC are sufficient in the absence of functional ligands on dendritic cells as the immune response proceeds.

\section{DISCUSSION}

In these studies, we establish that Notch signaling in CD4+ T cells represents an important checkpoint in the bifurcation between Tfh cell- and Th2 cell-driven hallmarks of type-2 immunity. Notch receptors 1 and 2 are required for Th cell generation, but are largely dispensable for Th2 differentiation in response to parasitic helminth infection. The importance of Notch in this bifurcation is reflected in the biology, as Tfh-mediated type-2 humoral hallmarks, such as IgE production, were impaired, while Th2orchestrated peripheral immunity, marked by worm clearance in the intestine and innate cell recruitment to the lung, remained intact. Furthermore, these studies reveal that Notch signaling is not only required for development and commitment of Th cells, but that Notch signals can enhance type- 2 cytokine production in committed Tfh cells. The enhanced cytokine production that results from increased NICD expression also appears specific to Tfh cells as lung-resident CD4+ T cells over-expressing NICD produced similar levels of IL-4 as CD4+ T cells-expressing endogenous NICD.
Many factors including BCL6, BATF, ASCL2, ICOS, and c-Maf have been deemed essential for Th cell development. ${ }^{21,22,39}$ These factors are part of a developmental circuit, which helps to determine Tfh cell fate. ${ }^{39}$ However, Notch has largely been left out of this conversation despite its requirement in Tfh development. ${ }^{11}$ Here, we show further evidence supporting a role for Notch in the Tfh developmental circuit, as inhibition of Notch signaling leads to decreased expression of c-Maf in Tfh cells. Of note, BATF, c-Maf, and RBP-J have all been reported to bind at the CNS2 enhancer of the IL-4 locus. Given the importance of the CNS2 region in the expression of IL-4 by Tfh cells, a likely mechanism as to how Notch signaling modulates the IL- 4 in Tfh cells is via NICD binding to RBP-J to modulate IL-4 expression. Whether and how RBP-J and NICD interact with c-Maf and BATF at this site is not known. As such, further work investigating how Notch selectively mediates cell fate and influences type-2 cytokine potential in the context of these other factors will be an important next step in understanding Th cell generation and function.

Two competing models for Notch in Th1, Th2, and Th17 cell differentiation have emerged, and are also likely at play in Tfh cell fate determination. The unbiased amplifying or facilitating model implies that Notch signals potentiate an already established cytokine potential in developing subsets. ${ }^{17}$ The instructive model suggests that the signals from the engagement of different Notch ligands by Notch receptors can direct 

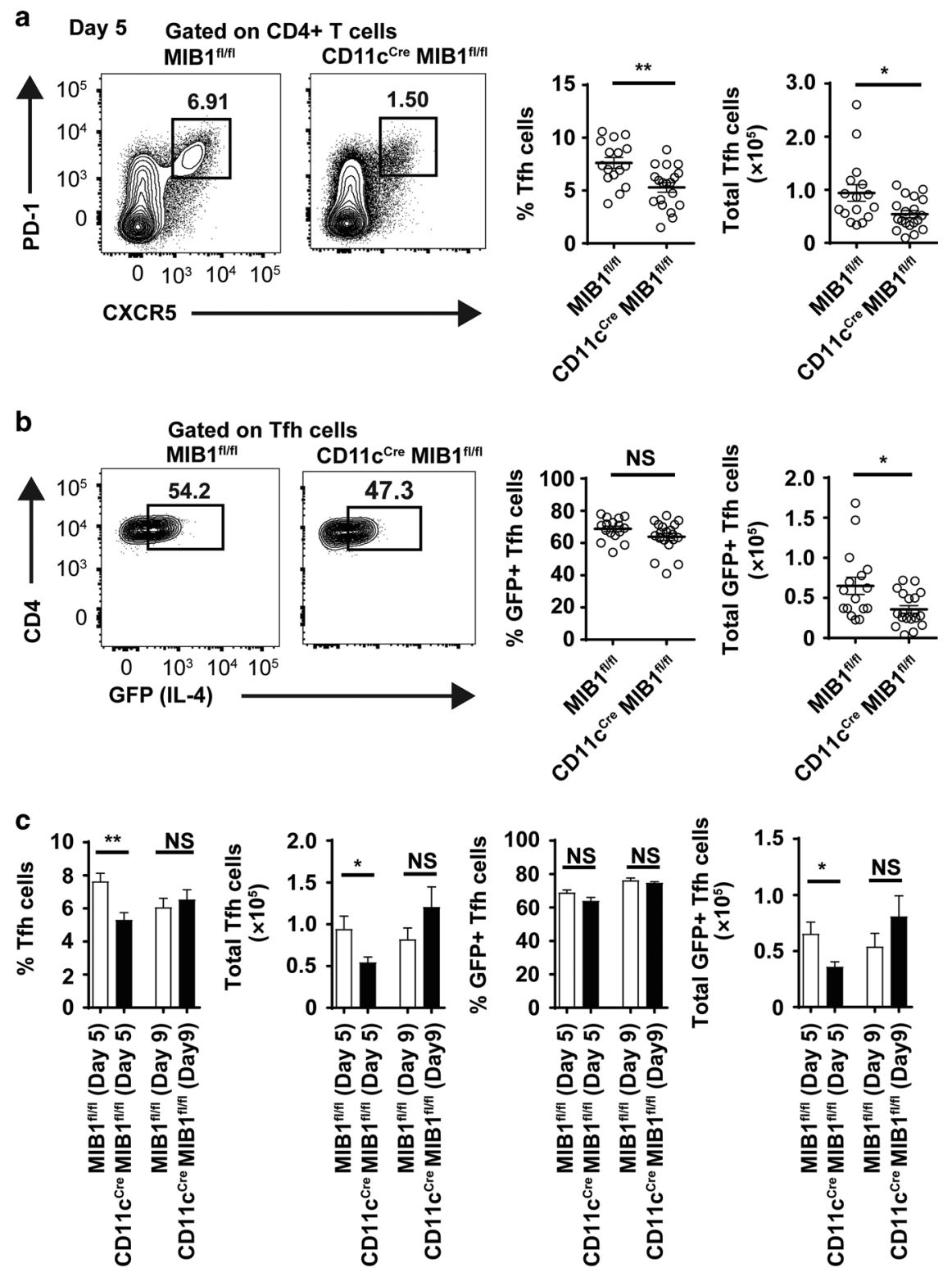

Fig. 7 Deletion of functional Notch ligands on conventional dendritic cells is important in early Tfh cell fate decision. IL4 ${ }^{4 g e t} C D 11 c^{\text {Cre }}$ Mib1 ${ }^{\text {fl/fl }}$ $(n=19)$ and IL $4^{4 g e t} \operatorname{Mib}^{f / f l}(n=16)$ mice were infected with $N$. brasiliensis and mediastinal lymph nodes were harvested 5 days later for flow cytometry. a Representative contour plots of CD4+ T cells. Gates indicate the percentage of Tfh (PD1+CXCR5+) cells. Graphs show quantification of percent and total number of Tfh cells. b Contour plots of Tfh cells. Gates indicate the percent of GFP+ Tfh cells from (a). Graphs show percent and total number of IL-4-expressing Tfh cells. (c) Graphs show percentage and total number of Tfh cells and IL-4expressing Tfh cells from IL $4^{4 g e t} C D 11 c^{\text {Cre }}$ Mib1 ${ }^{\text {fl/fl }}$ (filled bars; $n=11$ day $5, n=10$ day 9) and IL $4^{4 \text { get }}$ Mib1 ${ }^{\text {fl/fl }}$ (open bars; $n=19$ day 5 , $n=$ 16 day 9) 5 days and 9 days post helminth infection. Error bars represent $+/-$ SEM. Data shown is combined from three (day 9 ) or four (day 5 ) independent experiments with $\mathrm{n}=3-5$ mice per group. ${ }^{*} \mathrm{P}<0.05{ }^{* *} \mathrm{P}<0.01$ (unpaired two-tailed t-test)

differentiation toward a specific cell fate. ${ }^{13}$ Importantly, the results herein support aspects of both amplifying and instructive mechanisms in Notch-driven Tfh cell fate and function. As discussed above, over-expression of NICD showed that Notch signaling enhances IL-4 production in Tfh, but not Th2 cells. Furthermore, over-expression of NICD had no effect on driving GATA-3 expression in lung CD4 $+\mathrm{T}$ cells, while it did lead to enhanced expression of BCL6 in lymph node CD4+ T cells. The selective dependence on Notch ligands in Tfh vs. Th2 cell generation also reveals the potential presence of an instructivebased mechanism for Notch in Tfh cell fate choice. In its simplest form, the presence of functional Notch ligands promotes Tfh cell fate, but has a significantly reduced influence on Th2 cell development. This is not to say that Notch does not have a role in GATA3 expression or in amplifying Th2 cytokine competency, but rather that Th2 cells do not rely on Notch signals in vivo to the same extent as Tfh cells to achieve their ultimate effector fate. It is likely that Th2 cell fate is cemented and less influenced by Notch signals once a threshold of GATA-3 expression is achieved in developing Th2 cells. The presence of other IL- 4 and IL-13 producers in the lung can reinforce the Th2 phenotype and maintenance of GATA-3 in Th2 cells, potentially making the role of Notch in these settings less critical for driving Th2 responses in the periphery. While we did not see major defects in peripheral Th2 responses in mice with Notch-deficient $T$ cells, we did observe an influx of non-Th2 CD4+ T cells in the lungs of these mice. Given that Notch has been shown to influence fate and function in Th1, Th2, and Th17 cells, future work 


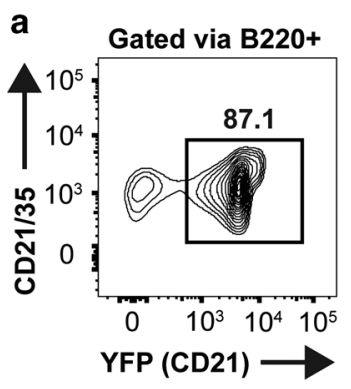

b Gated via CD45- B220- CD4-
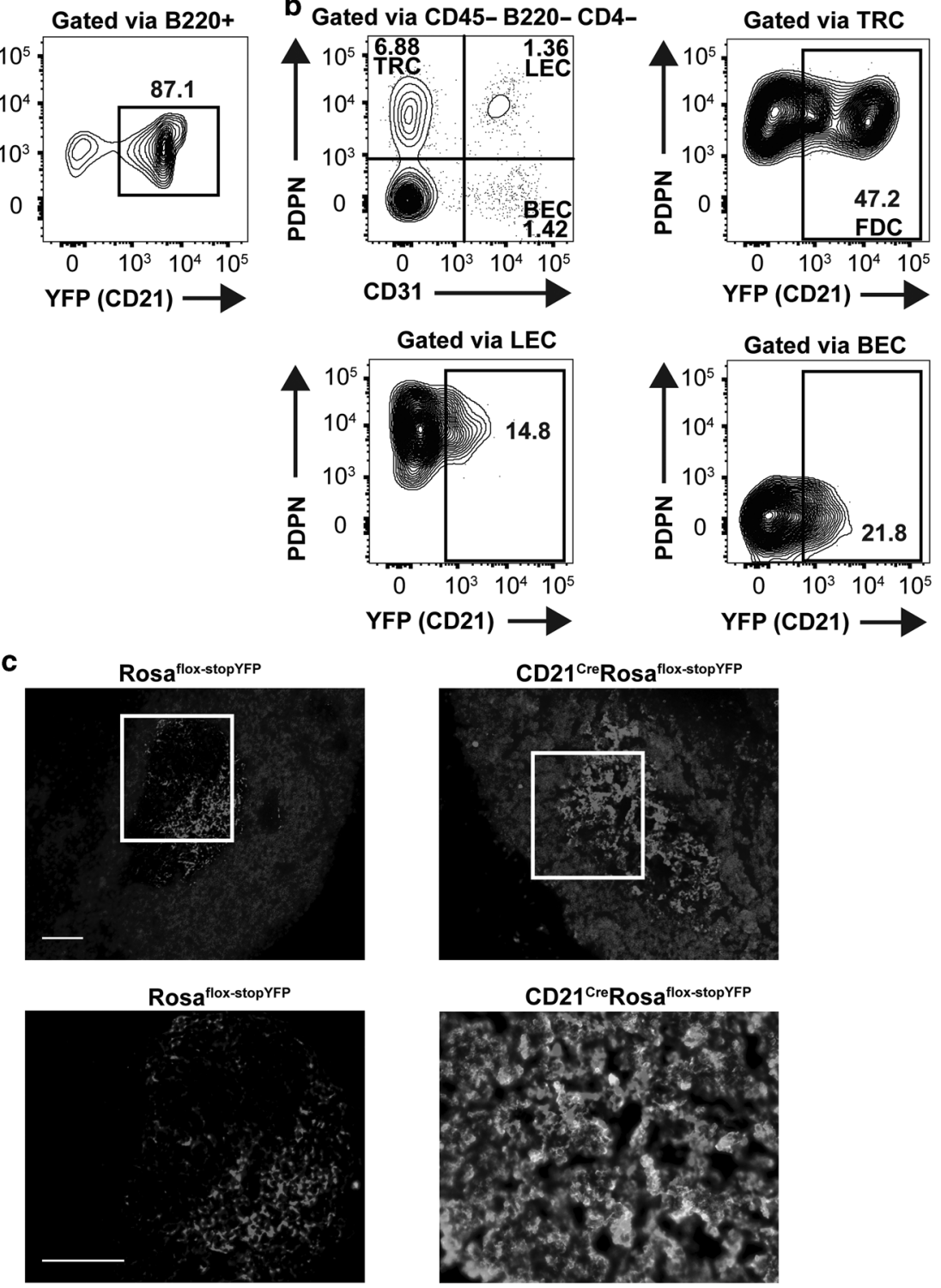

FDCM2 IgD YFP

Fig. 8 CD21 expression among lymph node stromal cell populations. CD21 $1^{\text {cre }}$ Rosa26 $6^{\text {Stop-flox-YFP }}$ and Rosa26 ${ }^{\text {Stop-flox-YFP }}$ mice were immunized with OVA emulsified in alum. Five days later inguinal, axial, and brachial lymph nodes were harvested and combined. The CD45- population was enriched and prepared for flow cytometry analysis. a Representative flow plot of the B220+ population. Gate marks YFP+ cells. b Contour plots from pooled lymph node samples gated on CD45-, B220-, and CD4-. Gates represent YFP expression on lymph node stromal cell populations: T-zone reticular cells (TRC; PDPN+, CD31-, CD21+), lymphatic endothelial cells (LEC; PDPN+ CD31+), and blood endothelial cells (BEC; PDPN- CD31+). FDCs are identified as YFP (CD21)-expressing TRCs. Data are representative of three independent experiments with $n=$ 2-3. c Nine days post subcutaneous footpad immunization with OVA/alum, immunofluorescence microscopy was performed on popliteal lymph nodes harvested from CD21 ${ }^{\text {cre }}$ Rosa26 $6^{\text {Stop-flox-YFP }}$ and Rosa26 Stop-flox-YFP mice. Sections were stained for IgD (blue), YFP (green), and FDCM2 (red). A composite image of IgD and FDCM2 stains is shown at the top of the panel (10x objective). A higher magnification (20x objective) of these images (area denoted by a white square) was taken and a composite image of FDCM2 and YFP is shown at the bottom of the panel. Scale bars (bottom left) represent $100 \mu \mathrm{m}$

investigating the effector fate and transcriptional program of these non-Th2 cells is of great interest.

The near complete absence of Tfh cells and $\lg \mathrm{E}$, despite normal Th2 development and peripheral type-2 immunity in mucosal tissues observed in this study is intriguing given conclusions made by prior studies. Several past studies looking at the necessity of Notch in type-2 immune responses have described Notch signaling to be essential for Th2 biology. ${ }^{40}$ These prior studies largely based their conclusions on in vitro Th2 polarization assays, ex vivo restimulation of lymph node resident CD4+ T cells, or used serum cytokines and $\lg E$ as surrogates for Th2 function. We now know that these readouts are not particularly good indicators of true Th2 cell biology in vivo. For example, IgE is dependent on Tfhderived IL-4 not type-2 cytokines generated by Th2 cells. ${ }^{4}$ Thus, prior studies designed to uncover a role for Notch in Th2 cell biology may have instead identified unappreciated defects in Tfh cells. We believe the experiments shown herein help to reconcile prior results and place them in a more unifying context.

The findings shown herein are of particular interest in light of previous studies investigating the role of Notch during Trichuris muris infection, another nematode infection model that requires Th2 cells for parasite expulsion. ${ }^{17,41}$ While in the $N$. brasiliensis 
a

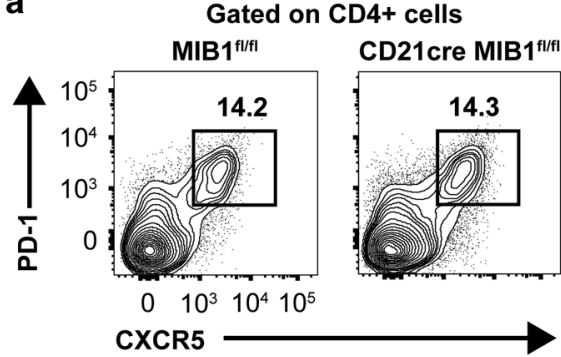

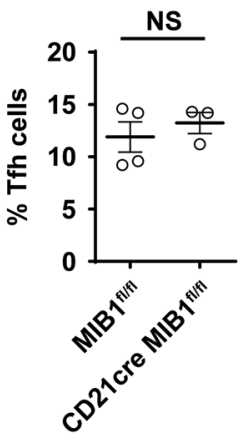

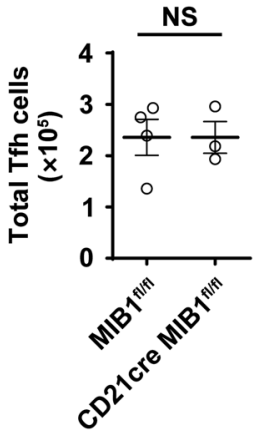

b

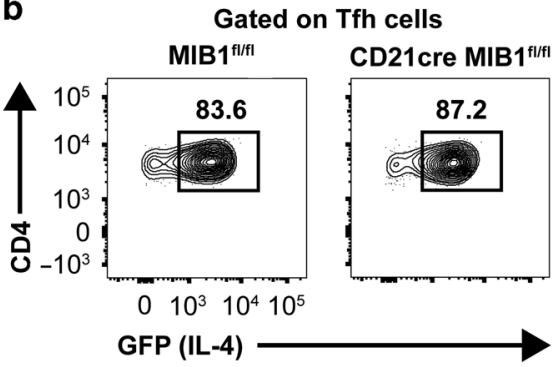

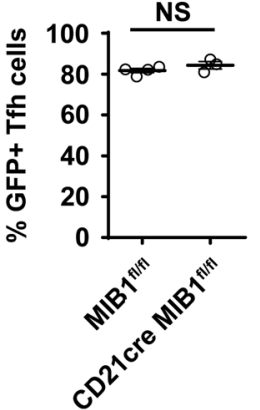

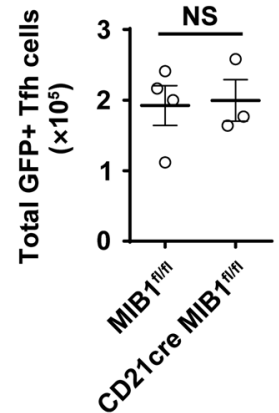

Fig. 9 Deletion of functional Notch ligands on FDCs and B cells is dispensable for Tfh differentiation and function. IL4 $4^{4 \mathrm{get}} \mathrm{CD} 21^{\mathrm{Cre}} \mathrm{Mib} 1^{\mathrm{f} / \mathrm{fl}}$ ( $n=$

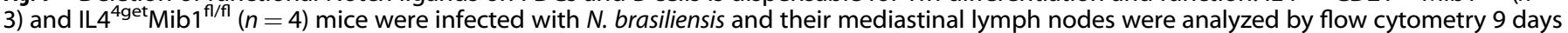
post infection. a Representative contour plots of CD4+ T cells. Gate represents Tfh (PD1+, CXCR5+) cells. Graphs show percent and total number of Tfh cells. b IL-4 expression was assessed and quantified in Tfh cell populations gated in panel a. Gate represents GFP + (IL-4 competent) Tfh cells. Error bars represent $+/$ - SEM. Data shown is representative of two independent experiments with $n=3-4$ mice per group

model we saw normal worm clearance but a complete loss of lgE when T cells lacked Notch1 and Notch2 suggesting normal Th2 function but impaired Tfh function, the T. muris studies showed both a loss of IgE and impaired worm expulsion when T cells expressed a dominant negative form of the co-activator mastermind/MAML (DNMAML), which is considered a pan-Notch inhibitor. This might at first pass suggest that both Tfh and Th2 cells are impaired using the condition DNMAML system. However, the authors show that administration of anti-CD3 to activate T cells in $T$. muris infected mice elicited no significant change in IL4, IL-5, and IL-13 in the serum compared to wild-type. This is consistent with Th2 differentiation and type-2 cytokine competency remaining largely intact in this setting. It will be interesting to confirm this by directly assessing Tfh cell and Th2 cell development in this model. Taken together, the data suggests that Notch signaling plays a less critical role in Th2 compared to Tfh differentiation, but supports a role for Notch in amplifying and optimizing Th2 function.

Another interesting area of investigation will be to determine why inhibition of Notch signaling seems to impair IL-4-mediated antibody isotypes more readily than interferon (IFN)-gammadriven isotype-switching. ${ }^{19,41,42}$ One might expect Notch to affect both IL-4-producing and IFN-gamma-producing Tfh cells equally as both are found in the germinal centers and affect isotypeswitiching. ${ }^{4}$ In support of Notch being involved in the generation of Th cells in both Th2 and Th1 settings, chronic Leishmania infection models show that Notch1 and Notch2-deficiency in $T$ cells leads to diminished Tfh cells and Tfh-dependent germinal center formation even in settings of robust type- 1 responses. Of note, $L$. major infection of CD4 ${ }^{\text {cre }}$ Notch $1 / 2^{\mathrm{f} / \mathrm{fl}}$ mice did not affect Th2 cell generation as Notch1/Notch2-deficiency in $T$ cells promoted lesion development on the normally resistant C57BL/6 background..$^{43}$ Lesion development is dependent on the presence of IL-4-producing Th2 cells. However, these findings do suggest that Th1 cell function was impacted in the absence of Notch signaling as their presence would prevent lesion development in this model.

Curiously, blocking of IFN- $\gamma$ seems to rescue $\operatorname{lgE}$ and $\operatorname{lgG} 1$ production in $T$. muris infected mice expressing a dominant negative form of MAML in T cells. Whether IFN-gamma is impacting Tfh cells directly in this case to promote lgE is not clear. It is likely that IFN-gamma from Th1 cells is suppressing systemic levels of type- 2 cytokines derived from Th2 cells during chronic helminth infection. Once inhibited by antibody, IFNgamma can no longer suppress Th2 function allowing systemic IL4 and non-Tfh-dervied IL-4 to influence $B$ cells. ${ }^{44,45}$ This finding, along with the data discussed above, may also suggest the presence of a non-canonical Notch signaling pathway that differentially involves MAML during Th1, Th2, and Tfh cell generation. Investigating these possibilities will be important for future studies.

The unexpected finding that Th2 cells were less dependent on Notch signals than their IL-4-producing Tfh cell counterparts suggested that these two cell subsets may also require distinct Notch ligands. One such bifurcating-ligand maybe DLL4. Inhibition of DLL4 promotes Th2 cell function in settings of allergic airway inflammation. ${ }^{46,47}$ The inhibitory role for DLL4 on Th2 cells is particularly intriguing given that DLL4 serves a promoting role in Th cell differentiation. ${ }^{48}$ When placed in the context of the data presented here, it is consistent with DLL4 acting as a switch for Tfh cell differentiation. In its absence, responding IL-4 competent cells may default toward a Th2 cell fate. DLL4 acting as a specific trigger for Tfh cells would also be consistent with our Mib1 results, as Mib1 has the highest affinity for DLL4 and is known to play a key role in DLL4-mediated Notch1 signaling during T-cell lineage development. ${ }^{49,50}$ 
How and if Notch signals complement TCR signals to direct Tfh cell fate is an interesting area for future investigation. The affinity of the TCR for antigen-MHC complexes and the dwell time spent on antigen presenting cell, are key factors in ultimately determining Tfh cell fate. ${ }^{51,52}$ However, additional signals likely influence Tfh cell fate either by increasing dwell time, and/or tuning TCR signals, or by independently promoting a Tfh-specific gene circuit in parallel to TCR signaling. ${ }^{39}$ In support of a complementary or parallel role for Notch in TCR-mediated differentiation of Tfh cells, previous studies have shown that TCR signaling increases Notch receptor expression and activation, and that Notch is required for TCR-mediated activation and proliferation of peripheral T cells. ${ }^{53,54}$ It is also clear that Notch signals can potentiate or inhibit TCR signals in the thymus and the periphery to regulate T-cell fate. ${ }^{55,56}$

The advantage of using Mib1 deletion is that it disrupts downstream signaling from all Notch ligands. Thus, it provides an unbiased approach to assess the involvement of Notch ligands in biology. However, as discussed above with regard to DLL4 in Th2 and Tfh cell generation, this prevents investigation into the individual roles specific Notch ligands might play in type-2 immunity. It is also unable to assess a role for non-canonical Notch ligands as Mib1 may not disrupt non-canonical Notch signaling. Despite these caveats, our findings do show that $\mathrm{CDCs}$ provide an important early source of Notch ligands. However, functional Notch ligands on CDC are not necessary to establish full Tfh cell effector potential. Similarly, B cells, T cells, and FDC, which interact frequently with Tfh cells in the B-cell follicles and germinal centers, are not required sources of Notch ligands for Tfh cell commitment. These findings help to further support a fibroblastic cell source of DLL4 as critical for Tfh cell commitment. ${ }^{48}$ Although initially suggested to be $\mathrm{FDC}^{48}$ our data indicates that other stromal sources are sufficient for Th commitment. Notch ligands on CDC, FDC, and B cells may contribute to the Tfh phenotype, but other (likely stromal sources) of Notch ligands can compensate in their absence.

This study places Notch in the company of BCL6 and ASCL2 as factors that selectively regulate $\mathrm{Thh}$ cell development and function relative to Th2 cells. However, unlike BCL6 and ASCL2, which work intracellularly to control Tfh cell fate, Notch and its ligands are present at the cell surface. This suggests that Notch and/or its ligands could represent important targets for immune-mediated therapies such as biologics focused on Tfhmediated disease outcomes, including allergies and other lgEdriven pathology.

\section{MATERIALS AND METHODS}

Mice

C57BL/6 Notch- $1^{\mathrm{f} / \mathrm{fl}}$ mice, ${ }^{57}$ Notch-2 ${ }^{\text {fl/fl } 58} \mathrm{CD} 4^{\text {cre }} 59$ Rosa26 $6^{\text {flox-stop- }}$ YFP 60 CD21 $1^{\text {cre } 61}$ Rosa26 ${ }^{\text {Stop-flox-NICD-GFP } 28}$ ER $^{\text {cre' } 62}$ and C57BL/6 mice were purchased from Jackson laboratories. MIB1 $1^{\mathrm{fl} / \mathrm{fl}}$ were generated by Young-Yun Kong (Pohang University of Science and Technology) and rederived at Duke University. ${ }^{35}$ CD11 $c^{\text {cre }}$ mice were provided by Gianna Hammer (Duke University). ${ }^{34} \mathrm{IL}-4^{4 g e t}$ and $\mathrm{IL}-4^{\mathrm{KN} 2}$ mice were provided by Richard Locksley (UCSF). ${ }^{20,27}$ OT-II mice were provided by Weiguo Zhang (Duke). All mice were housed in pathogen-free facilities following guidelines set by the Division of Laboratory Animal Resources, Duke University Medical Center, Biological Resource Center at National Jewish Health, and the Institutional Animal Care and Use Committee.

\section{Infections and worm counts}

$N$. brasiliensis was prepared as previously described. ${ }^{63}$ Mice were injected subcutaneously in the rear flank with 500 L3 larvae in saline solution. To conduct worm counts, small intestines were removed, opened longitudinally, and incubated in HBSS at $37^{\circ} \mathrm{C}$ for $1-2 \mathrm{~h}$. Adult worms were manually enumerated.
Notch inhibition studies

Where described, mice were immunized subcutaneously with 100 $\mu \mathrm{g}$ of OVA emulsified in alum in the footpad. On days 7, 8, and 9 post immunization mice were given GSI, Dibenzazepine (Selleckhem) intraperitoneally. The popliteal lymph node was harvested on day 10 for subsequent flow cytometric analysis.

\section{Tamoxifen injections}

Tamoxifen (T5648; Sigma) was diluted in USP grade corn oil. Two milligrams of tamoxifen was injected intraperitoneally once every $24 \mathrm{~h}$ for 3 days prior to infection.

\section{Cell proliferation study}

OT-II CD4+ T cells were isolated and stained with $5 \mu \mathrm{M}$ eFluor670 proliferation dye (65-0840-85; Thermo Fisher). In all, $1.5 \times 10^{6}$ OT-II T cells were then transferred I.V. into the mice described. Twentyfour hours post transfer the mice were immunized by subcutaneous injection of $100 \mathrm{ug}$ of OVA emulsified alum in the footpad. Three days post immunization, the popliteal lymph nodes were harvested to assess proliferation of the transferred cells.

Immunofluorescence histology

Lymph nodes were fixed with 4\% PFA for $2 \mathrm{~h}$, incubated in $30 \%$ sucrose, and frozen in optimal cutting temperature compound (O.C.T.). Lungs were inflated with, then incubated in 4\% PFA for 3 $\mathrm{h}$, and incubated in $30 \%$ sucrose prior to freezing. Eight micron sections were cut and epitopes on slides were stained using tyramide amplification as described. ${ }^{21}$ eGFP and YFP were detected using a purified polyclonal rabbit anti-mouse GFP (NB600-308; Novus Biologicals) followed by biotinylated $F\left(a b^{\prime}\right)_{2}$ donkey anti-rabbit (Jackson ImmunoResearch Laboratories). Biotinylated anti-mouse CD4 (RM4-5; Biolegend); IgD (11-26c; eBiosciences), and FDCM2 (212-MK-2FDCM2; ImmunoKontact) were used to detect CD4 T cells, B cells, and FDC, respectively. 4',6-Diamidine-2'-phenylindole dihydrochloride (DAPl; $0.5 \mu \mathrm{g} / \mathrm{ml}$; Roche) in PBS was used to counterstain nuclei prior to mounting on coverslips with Vectashield (Vector laboratories). Images were collected with a Zeiss Axio Imager. Analysis of images was performed using FIJI (ImageJ) software.

\section{Flow cytometry}

Lungs were chopped with a razor blade, digested with $250 \mu \mathrm{g} /$ $\mathrm{ml}$ Collagenase XI (C7657; Sigma), $50 \mu \mathrm{g} / \mathrm{ml}$ Liberase (145495; Roche), $1 \mathrm{mg} / \mathrm{ml}$ Hyaluronidase (h3506; Sigma), and $200 \mu \mathrm{g} / \mathrm{ml}$ DNase I (DN25; Sigma) in RPMI 1640 to prepare single-cell suspensions. Single-cell suspensions of lymph nodes were prepared by mechanical dissociation. Surface stains were performed with the following antibodies in 2\% FCS in PBS: APC/Cy7 conjugated to anti-mouse CD4 (RM4-5); PerCP-Cy5.5 conjugated to anti-mouse CD11C (N418), anti-mouse CD4 (RM45), anti-mouse CD8 (53-6.7), anti-mouse/human CD45R/B220 (RA3-6B2), anti-mouse CD31 (390); phycoerythrin (PE)/Cy7 conjugated to anti-mouse CD279/PD-1 (RMP1-30), CD49b (DX5), and anti-mouse CD21/35 (7E9); Alexa Fluor 647 conjugated to anti-mouse/human GL7 (GL7); Allophycocyanin (APC) conjugated to anti-mouse PDPN/GP38 (8.1.1); and PEconjugated to anti-mouse Notch 1 (HMN1-12), anti-mouse Notch2 (16F11), anti-mouse SiglecF (E50-2440), anti-mouse CD131 (JORO50), streptavidin from Biolegend. PE or APC conjugated to anti-human CD2 (S5.5) from Invitrogen. APCeFluor 780 conjugated to anti-human/mouse CD45R/B220 (RA36B2) from eBioscience. PE/Cy7 conjugated to anti-mouse CD95 (JO2) and biotinylated anti-rat/mouse CD185/CXCR5 (2G8) from BD biosciences. Cells were resuspended in $2 \%$ FCS in PBS containing DAPI $(0.5 \mu \mathrm{g} / \mathrm{ml})$. Lymphocyte and singlet gates were performed by size and granularity based on forward and side scatter. Live cells were gated based on DAPI exclusion. Data was 
collected on a FACSCanto II or LSR II (BD Biosciences) cytometer and analyzed using FlowJo (TreeStar).

Transcription factor staining

Surface stains were performed followed by staining of dead cells with Fixable Violet Dead Cell Stain kit (L34964; Thermo Fisher). A Foxp3 transcription factor staining buffer kit (eBioscience) was used to fix and permeabilize cells. Finally, these antibodies were used for transcription factor staining PEconjugated to anti-mouse/human GATA-3 (TWAJ; eBioisciences), anti-mouse/human CMAF (SymOF1; eBiosciences), anti-mouse/ human IRF4 (3E4; eBiosciences), anti-mouse/human BATF (9B5A13); and Alexa Fluor 647 conjugated to anti-mouse BCL6 (K112-91; BD Biosciences).

\section{FDC isolation}

CD $21^{\text {cre }}$ Rosa $26^{\text {Stop-flox-YFP }}$ and Rosa $26^{\text {Stop-flox-YFP }}$ mice were immunized subcutaneously with OVA emulsified in Imject alum (Thermo Fisher) in both rear flanks and upper back. Each mouse received a total of $100 \mu \mathrm{g}$ of OVA/alum. Mice were sacrificed and axial, brachial, and inguinal nodes were harvested. Lymph nodes were opened using $26 \mathrm{G}$ needles and digested at $37^{\circ} \mathrm{C}$ for $30 \mathrm{~min}$ with Collagenase IV (1 mg/ml; 17104-019; Gibco); DNase I $(40 \mu \mathrm{g} / \mathrm{ml}$; DN25; Sigma) in RPMI 1640. Pipetting was performed every $10 \mathrm{~min}$ to assist in digestion of the tissue. After incubation, $5 \mathrm{mM}$ EDTA (03690; Sigma) was added to the digestion to help dissociate cell aggregates. Cells were filtered through an $80 \mu \mathrm{m}$ mesh. StemCell RapidSpheres (19860 A) kit was used to positively select CD45+ cells using biotinylated anti-mouse CD45 (30F11; Biolegend). Flow through, highly enriched for CD45- cells, was taken and stained for flow cytometry.

To image FDCs by immunofluorescence histology, $C D 21{ }^{\text {cre }} \mathrm{R}$ osa $26^{\text {Stop-flox-YFP }}$ and Rosa26 $6^{\text {Stop-flox-YFP }}$ mice were immunized subcutaneously in the footpad with $100 \mu \mathrm{g}$ OVA emulsified in alum. The popliteal lymph node was harvested 9 days later and prepared for histologic analysis. Staining for FDCM2 was performed to specifically identify FDCs.

Enzyme-linked immunosorbent assay (ELISA)

Ninety-six well plates were coated with rat anti-mouse lgE (R35-72; BD Bioscience) and blocked using 5\% BSA. Serum samples were added and total lgE detected using biotinylated antimouse IgE (R35-118; BD Biosciences) followed by streptavidinHRP and o-phenylenediamine. An EMax Precision Microplate Reader (Molecular Devices) was used to quantify total IgE concentrations based on purified IgE (MEB-38; Biolegend) standard curves.

\section{Real-time PCR}

$\mathrm{CD} 11 \mathrm{c}+\mathrm{MHCll}+\mathrm{DCs}$ were sorted with a BD FACSAria Fusion or Astrios (Beckman Coulter) and resuspended in TRlzol reagent (15596026; Thermo Fisher). Chloroform and ethanol precipitation were used to isolate RNA from the samples. DNA was eliminated using a DNase I kit (18068015; Thermo Fisher). cDNA was synthesized by reverse transcription (18080051; Thermo Fisher) per kit instructions with oligo (dT) primers followed by RNase $\mathrm{H}$ treatment. Real-time PCR was performed on the CDNA using an allin-one SYBR green qPCR mix (QP001-01; GeneCopoeia) amplified with primers against Mind bomb1 (QT00110453; Qiagen) and $\beta$-actin (QT00519526; Qiagen) on an Applied Biosystems StepOnePlus RT PCR system. Expression of Mind bomb1 was calculated relative to $\beta$-actin.

\section{Statistics}

Two-tailed paired or unpaired $t$-tests were performed. $P$-values $<0.05$ and $>0.01$ are indicated with a single asterisk $(*), P$-values $<0.01$ and $>0.001$ are indicated with double asterisk $\left(^{* *}\right)$, and $P$ values $<0.001$ are indicated with triple asterisk $\left.{ }^{* * *}\right)$.

\section{ACKNOWLEDGEMENTS}

We thank Richard Locksley for providing the IL $4^{4 g e t}$ and IL4 ${ }^{\mathrm{KN} 2}$ reporter strains, Gianna Hammer for providing the CD11 $c^{\text {cre }}$ strain, Young-Yun Kong for allowing us to use the $\mathrm{MIB}^{\mathrm{fl} / \mathrm{fl}}$ mice, and Ann Miller for general technical assistance. Additionally, we would like to thank the Duke Light Microscopy Core Facility, the Duke Flow Cytometry Facility, and the National Jewish Health Flow cytometry core. This work was supported by the National Institutes of Health Grant Al119004 (R.L.R.).

\section{AUTHOR CONTRIBUTIONS}

M.D. and R.L.R conceived the work, designed the study, and wrote the manuscript. M. D. performed the experiments and analyzed the data. R.L.R supervised the study.

\section{ADDITIONAL INFORMATION}

The online version of this article (https://doi.org/10.1038/s41385-018-0012-9) contains supplementary material, which is available to authorized users.

Competing interests: The authors declare no competing interests.

Publisher's note: Springer Nature remains neutral with regard to jurisdictional claims in published maps and institutional affiliations.

\section{REFERENCES}

1. Locksley, R. M. Asthma and allergic inflammation. Cell 140, 777-783 (2010).

2. Pulendran, B. \& Artis, D. New paradigms in type 2 immunity. Science 337, 431-435 (2012).

3. Bao, K. \& Reinhardt, R. L. The differential expression of IL-4 and IL-13 and its impact on type-2 immunity. Cytokine 75, 25-37 (2015).

4. Reinhardt, R. L., Liang, H. E. \& Locksley, R. M. Cytokine-secreting follicular T cells shape the antibody repertoire. Nat. Immunol. 10, 385-393 (2009).

5. Snapper, C. M. \& Paul, W. E. Interferon-gamma and B cell stimulatory factor-1 reciprocally regulate Ig isotype production. Science 236, 944-947 (1987).

6. Liang, H. E. et al. Divergent expression patterns of IL-4 and IL-13 define unique functions in allergic immunity. Nat. Immunol. 13, 58-66 (2012).

7. Yusuf, I. et al. Germinal center T follicular helper cell IL-4 production is dependent on signaling lymphocytic activation molecule receptor (CD150). J. Immunol. 185, 190-202 (2010).

8. Harada, Y. et al. The $3^{\prime}$ enhancer CNS2 is a critical regulator of interleukin-4mediated humoral immunity in follicular helper T cells. Immunity 36, 188-200 (2012).

9. Vijayanand, P. et al. Interleukin-4 production by follicular helper T cells requires the conserved II4 enhancer hypersensitivity site V. Immunity 36, 175-187 (2012).

10. Tanaka, S. et al. The interleukin- 4 enhancer CNS-2 is regulated by Notch signals and controls initial expression in NKT cells and memory-type CD4 T cells. Immunity 24, 689-701 (2006).

11. Auderset, F. et al. Notch signaling regulates follicular helper $\mathrm{T}$ cell differentiation. J. Immunol. 191, 2344-2350 (2013).

12. Radtke, F., MacDonald, H. R. \& Tacchini-Cottier, F. Regulation of innate and adaptive immunity by Notch. Nat. Rev. Immunol. 13, 427-437 (2013).

13. Amsen, D. et al. Instruction of distinct CD4 T helper cell fates by different notch ligands on antigen-presenting cells. Cell 117, 515-526 (2004).

14. Maekawa, Y. et al. Delta1-Notch3 interactions bias the functional differentiation of activated CD4+ T cells. Immunity 19, 549-559 (2003).

15. Krawczyk, C. M., Sun, J. \& Pearce, E. J. Th2 differentiation is unaffected by Jagged2 expression on dendritic cells. J. Immunol. 180, 7931-7937 (2008).

16. Worsley, A. G. et al. Dendritic cell expression of the Notch ligand jagged2 is not essential for Th2 response induction in vivo. Eur. J. Immunol. 38, 1043-1049 (2008).

17. Bailis, W. et al. Notch simultaneously orchestrates multiple helper T cell programs independently of cytokine signals. Immunity 39, 148-159 (2013).

18. Ong, C. T., Sedy, J. R., Murphy, K. M. \& Kopan, R. Notch and presenilin regulate cellular expansion and cytokine secretion but cannot instruct Th1/Th2 fate acquisition. PLOS ONE 3, e2823 (2008).

19. Amsen, D. et al. Direct regulation of Gata3 expression determines the $T$ helper differentiation potential of Notch. Immunity 27, 89-99 (2007).

20. Mohrs, M., Shinkai, K., Mohrs, K. \& Locksley, R. M. Analysis of type 2 immunity in vivo with a bicistronic IL-4 reporter. Immunity 15, 303-311 (2001).

21. Bao, K. et al. BATF modulates the Th2 locus control region and regulates CD4+ T cell fate during antihelminth immunity. J. Immunol. 197, 4371-4381 (2016).

22. Sahoo, A. et al. Batf is important for IL-4 expression in T follicular helper cells. Nat. Commun. 6, 7997 (2015). 
23. Ise, W. et al. The transcription factor BATF controls the global regulators of classswitch recombination in both B cells and T cells. Nat. Immunol. 12, 536-543 (2011).

24. Bollig, N. et al. Transcription factor IRF4 determines germinal center formation through follicular T-helper cell differentiation. Proc. Natl Acad. Sci. USA 109, 8664-8669 (2012).

25. Andris, F. et al. The transcription factor c-Maf promotes the differentiation of follicular helper T cells. Front. Immunol. 8, 480 (2017).

26. Kroenke, M. A. et al. Bcl6 and Maf cooperate to instruct human follicular helper CD4 T cell differentiation. J. Immunol. 188, 3734-3744 (2012).

27. Mohrs, K., Wakil, A. E., Killeen, N., Locksley, R. M. \& Mohrs, M. A two-step process for cytokine production revealed by IL-4 dual-reporter mice. Immunity 23, 419-429 (2005).

28. Murtaugh, L. C., Stanger, B. Z., Kwan, K. M. \& Melton, D. A. Notch signaling controls multiple steps of pancreatic differentiation. Proc. Natl Acad. Sci. USA 100, 14920-14925 (2003).

29. Fang, T. C. et al. Notch directly regulates Gata3 expression during T helper 2 cell differentiation. Immunity 27, 100-110 (2007).

30. Goenka, R. et al. Cutting edge: dendritic cell-restricted antigen presentation initiates the follicular helper $\mathrm{T}$ cell program but cannot complete ultimate effector differentiation. J. Immunol. 187, 1091-1095 (2011).

31. Barnett, L. G. et al. B cell antigen presentation in the initiation of follicular helper T cell and germinal center differentiation. J. Immunol. 192, 3607-3617 (2014).

32. Dahlgren, M. W. et al. T follicular helper, but not Th1, cell differentiation in the absence of conventional dendritic cells. J. Immunol. 194, 5187-5199 (2015).

33. Guo, B., McMillan, B. J. \& Blacklow, S. C. Structure and function of the Mind bomb E3 ligase in the context of Notch signal transduction. Curr. Opin. Struct. Biol. 41, 38-45 (2016).

34. Caton, M. L., Smith-Raska, M. R. \& Reizis, B. Notch-RBP-J signaling controls the homeostasis of CD8- dendritic cells in the spleen. J. Exp. Med. 204, 1653-1664 (2007).

35. Koo, B. K. et al. An obligatory role of mind bomb-1 in notch signaling of mammalian development. PLOS ONE 2, e1221 (2007).

36. Wang, $X$. et al. Follicular dendritic cells help establish follicle identity and promote B cell retention in germinal centers. J. Exp. Med. 208, 2497-2510 (2011).

37. Victoratos, P. et al. FDC-specific functions of p55TNFR and IKK2 in the development of FDC networks and of antibody responses. Immunity 24, 65-77 (2006).

38. Roozendaal, R. \& Carroll, M. C. Complement receptors CD21 and CD35 in humoral immunity. Immunol. Rev. 219, 157-166 (2007).

39. Crotty, S. T follicular helper cell differentiation, function, and roles in disease. Immunity 41, 529-542 (2014).

40. Amsen, D., Antov, A. \& Flavell, R. A. The different faces of Notch in T-helper-cell differentiation. Nat. Rev. Immunol. 9, 116-124 (2009).

41. Tu, L. et al. Notch signaling is an important regulator of type 2 immunity. J. Exp. Med. 202, 1037-1042 (2005).

42. Backer, R. A. et al. A central role for Notch in effector CD8(+) T cell differentiation. Nat. Immunol. 15, 1143-1151 (2014).

43. Auderset, F. et al. Redundant Notch1 and Notch2 signaling is necessary for IFNgamma secretion by $\mathrm{T}$ helper 1 cells during infection with Leishmania major. PLoS Pathog. 8, e1002560 (2012).

44. Perona-Wright, G., Mohrs, K. \& Mohrs, M. Sustained signaling by canonical helper T cell cytokines throughout the reactive lymph node. Nat. Immunol. 11, 520-526 (2010).

45. Turqueti-Neves, A. et al. B-cell-intrinsic STAT6 signaling controls germinal center formation. Eur. J. Immunol. 44, 2130-2138 (2014).

46. Jang, S., Schaller, M., Berlin, A. A. \& Lukacs, N. W. Notch ligand delta-like 4 regulates development and pathogenesis of allergic airway responses by modulating IL-2 production and Th2 immunity. J. Immunol. 185, 5835-5844 (2010).

47. Schaller, M. A. et al. Notch ligand Delta-like 4 regulates disease pathogenesis during respiratory viral infections by modulating Th2 cytokines. J. Exp. Med. 204, 2925-2934 (2007).
48. Fasnacht, N. et al. Specific fibroblastic niches in secondary lymphoid organs orchestrate distinct Notch-regulated immune responses. J. Exp. Med. 211 2265-2279 (2014).

49. Shah, D. K., Mohtashami, M. \& Zuniga-Pflucker, J. C. Role of recycling, Mindbomb1 association, and exclusion from lipid rafts of delta-like 4 for effective Notch signaling to drive T cell development. J. Immunol. 189, 5797-5808 (2012).

50. Luca, V. C. et al. Structural biology. Structural basis for Notch1 engagement of Delta-like 4. Science 347, 847-853 (2015).

51. Tubo, N. J. et al. Single naive CD4(+) T cells from a diverse repertoire produce different effector cell types during infection. Cell 153, 785-796 (2013).

52. Fazilleau, N., McHeyzer-Williams, L. J., Rosen, H. \& McHeyzer-Williams, M. G. The function of follicular helper $T$ cells is regulated by the strength of $T$ cell antigen receptor binding. Nat. Immunol. 10, 375-384 (2009).

53. Palaga, T., Miele, L., Golde, T. E. \& Osborne, B. A. TCR-mediated Notch signaling regulates proliferation and IFN-gamma production in peripheral $\mathrm{T}$ cells. $\mathrm{J}$. Immunol. 171, 3019-3024 (2003).

54. Guy, C. S. et al. Distinct TCR signaling pathways drive proliferation and cytokine production in T cells. Nat. Immunol. 14, 262-270 (2013).

55. Huang, Y. H., Li, D., Winoto, A. \& Robey, E. A. Distinct transcriptional programs in thymocytes responding to $T$ cell receptor, Notch, and positive selection signals. Proc. Natl Acad. Sci. USA 101, 4936-4941 (2004).

56. Eagar, T. N. et al. Notch 1 signaling regulates peripheral T cell activation. Immunity 20, 407-415 (2004).

57. Yang, X. et al. Notch activation induces apoptosis in neural progenitor cells through a p53-dependent pathway. Dev. Biol. 269, 81-94 (2004).

58. McCright, B., Lozier, J. \& Gridley, T. Generation of new Notch2 mutant alleles. Genesis 44, 29-33 (2006).

59. Sawada, S., Scarborough, J. D., Killeen, N. \& Littman, D. R. A lineage-specific transcriptional silencer regulates CD4 gene expression during $T$ lymphocyte development. Cell 77, 917-929 (1994).

60. Srinivas, S. et al. Cre reporter strains produced by targeted insertion of EYFP and ECFP into the ROSA26 locus. BMC Dev. Biol. 1, 4 (2001).

61. Kraus, M., Alimzhanov, M. B., Rajewsky, N. \& Rajewsky, K. Survival of resting mature $B$ lymphocytes depends on BCR signaling via the Igalpha/beta heterodimer. Cell 117, 787-800 (2004).

62. Ventura, A. et al. Restoration of p53 function leads to tumour regression in vivo Nature 445, 661-665 (2007).

63. Voehringer, D., Reese, T. A., Huang, X., Shinkai, K. \& Locksley, R. M. Type 2 immunity is controlled by IL-4/IL-13 expression in hematopoietic non-eosinophil cells of the innate immune system. J. Exp. Med. 203, 1435-1446 (2006).

(i) Open Access This article is licensed under a Creative Commons Attribution-NonCommercial-ShareAlike 4.0 International License which permits any non-commercial use, sharing, adaptation, distribution and reproduction in any medium or format, as long as you give appropriate credit to the original author(s) and the source, provide a link to the Creative Commons license, and indicate if changes were made. If you remix, transform, or build upon this article or a part thereof, you must distribute your contributions under the same license as the original. The images or other third party material in this article are included in the article's Creative Commons license, unless indicated otherwise in a credit line to the material. If material is not included in the article's Creative Commons license and your intended use is not permitted by statutory regulation or exceeds the permitted use, you will need to obtain permission directly from the copyright holder. To view a copy of this license, visit http://creativecommons.org/licenses/by-nc-sa/4.0/.

(c) The Author(s) 2018 